\title{
The Role of Saudi Woman in Achieving Intellectual Safety ${ }^{i}$
}

\author{
Norah Alotaibi \\ College of Social Services, Princess Nourah bint Abdulrahman University, Saudi Arabia
}

Copyright $(2017$ by authors, all rights reserved. Authors agree that this article remains permanently open access under the terms of the Creative Commons Attribution License 4.0 International License

\begin{abstract}
The present study is an attempt to identify the role played by Saudi woman in achieving intellectual safety in Saudi community. The study utilized social survey method and the questionnaire was applied to a sample, randomly selected, consisted of 1300 female university students from (Princess Nourah bint Abddulrahman University, King Saud University, and Imam Muhammad ibn Saud Islamic University) for the first semester of the academic year 2016/2017. The study showed that there is an agreement of the participants on the role of woman in raising children according to the culture of family dialogue and its mechanisms rated between 2.44 and 2.64, and the role of woman in consolidating of moderate Islamic values in the minds of children rated between 2.70 and 2.86. It also confirmed the role of woman in reinforcing human self of children, respect and development of children while maintaining cultural excellence rated between 2.51 and 2.69, and the role of woman in raising children to respect freedoms of others rated between 2.52 and 2.74. In light of the study finding, some recommendations have been made.
\end{abstract}

Keywords The Role of Woman, Intellectual Safety, Saudi Community

\section{Introduction}

Intellectual safety is an essential request for every nation to secure protection for the society, in general, and young people, in particular, especially in the Islamic countries from extremist thoughts. It is a religious obligation. It causes cohesion and unity of thought, method, and aim. If it is absent, there will be imbalance in all forms of security (Elbashry, 2000, p. 30). The family is the principal interface with individual and society. It is of great importance in forming the individual's personality who acquires responsibility towards others and living according to the rules imposed by the society (Elbaqmey, 2010, p. 49). By creating a family, Islam seeks to accomplish great objectives that cover all aspects of the society, greatly affect Muslims' life, and protect the community from chaos. It also accomplishes a spiritual aim, namely family creation is the best means of refinement of souls and upgrade morals that lead to making empathy, compassion, and selflessness (Elhazemy, 2010, p. 83). A woman plays a major role in the family and society as she is responsible for socialization that helps form the individual's personality, ideas, doctrines, and attitudes. She also affects the attitudes using positive methods. She, with the husband, can guide children's behavior; discuss with them; consolidate original and moderate values and cultural distinctiveness; equip them with the sound knowledge to protect their doctrines, and thought. It also protects them from being victims of wrong ideas and thoughts that distort thought, affects behavior, and cause committing crimes, violence, and other forms of intolerable misconduct. Therefore, many studies address the role of socialization institutions in achieving intellectual safety, especially the family and school.

For example, Ameen (2012) stresses that a family should depend on the diversity of educational methods, having dialogue with children, having a space of effective understanding with children, renouncing oppressive methods, and dealing with them in more appropriate ways according to the conditions of community. Elrashedy (2012) reported that environmental and social indicators are the greatest factors in creating intellectual extremism. He recommends activating the role of family and educational institution in educating young people, rejecting extremism, and creating a social environment free from intellectual extremism. Elhaweesh (2011) argues that socialization's institutions (family, school, and mosque) are among the most significant factors that reinforce moderation in their doctrines and actions to upgrade the skills of dialogue, freedom of opinions, democracy, and effective contribution in all community issues. Eljahny (2005) reports that family's function in reinforcing intellectual safety; the personality of the young people is formed via conduct methods practiced by their families. In addition, the type of conduct at home is reflected in the personality and behavior of children in future. Elhaedar (2002) argues that the family plays a major and consolidated role with community institutions in facing threats to intellectual safety. Greenword (1996) refers to the necessity of early intervention using crime's preventive programs is an active tool to prevent or reduce delinquency and criminal behavior. Grahmj and Bowling (1998) show the 
importance of designing a preventive program to protect students against dangerous situations. Sharman (1997) attempted to investigate the impact of an integrated curriculum to protect from crime. It covers programs of violence prevention, protecting against theft, and helping students violate law. Conrad (1995) affects students' awareness development of the local community problems. It also helps develop thinking and contemplation of their duties and rights. Yahyaei and Mosadegh Zadeh (2014) conclude that the mother plays a significant role as the first teacher in the child's life and the first one in charge of the family's intellectual safety. Egan (2007) refers to the importance of family and motherhood roles in treating the issues of woman involved in terrorist crimes.

\subsection{Statement of the Problem}

Currently, the Islamic world faces intellectual, political, and economic challenges and difficulties, particularly terrorism and extremism. They are mainly faced by intellectual safety because people live in security and feel safe of the components of originality, quality culture, and intellectual system through the sanity of thinking and understanding from the deviation of the religious and political issues and depicting the universe. Accordingly, we should unite to protect our homeland. Furthermore, a woman should believe that she plays an important role in accomplishing intellectual safety, that is an urgent need at the time, feel the importance of security in the society, and define the right path that should be followed mentally and intellectually. Total security, in the broad sense, is a general responsibility and each person should participate. Therefore, the woman has the largest part of the strong basis of the family and the first assistant, if not the real leader, of driving the family.

There is no doubt that the woman plays the key role in educating a generation on empathy, selflessness, modesty, honesty, bonds of kinship, cooperation in righteousness and piety, forgiveness, courage, filial piety, respect for the elderly, and respect for others' perspectives. She actually instills good morals in children till they become customs related to behaviors. They also acquire assuming responsibility and fulfilling the rights. Additionally, they are brought up on philanthropy to others regarding doing favor and advising in a sound way to others. One of the main family responsibilities, particularly for the woman, is protecting children from any intellectual deviation. That is, it is not only responsible for providing necessities to its members, but participating in resolving and solving difficulties. It also participates with other community institutions "school, mosque, media and security" in forming the correct concepts and creating a strong relation with the family to reinforce intellectual safety among young people, work on implanting patriotism, and maintain keeping its assets. It is responsible for creating an intellectual and secure reinforcement for children to protect them. Therefore, a
Saudi woman should believe that she has a key role in achieving intellectual safety, which is a must, and feel the importance of this role in the society. She should also realize her duties towards the country to face this wrong thought, stressing that achieving intellectual safety in the community is a shared responsibility. Hence, the problem of the current study has been defined in identifying the role played by Saudi woman in achieving intellectual safety in the Saudi community.

\subsection{Questions}

The study attempts to answer the following major question: What is the role of woman in achieving intellectual safety in the Saudi community?

It is further divided into the following minor questions:

- What is the role of woman in bringing up children according to the culture and mechanism of family dialogue?

- What is the role of woman in implanting moderate Islamic values in children?

- What is the role of woman in reinforcing and respecting the self and maintaining cultural distinctiveness?

- What is the role of woman in bringing up children on respecting freedom?

- What is the proposal of woman's role in achieving intellectual safety in the Saudi community?

\subsection{Objectives}

The study mainly attempts to identify the role of Saudi woman in achieving intellectual safety in the Saudi community. It also aims at:

1. Defining the role of woman in bringing up children according to the culture and mechanism of family dialogue.

2. Identifying the role of woman in implanting moderate Islamic values in children.

3. Identifying the role of woman in reinforcing and respecting the self while maintaining cultural distinctiveness.

4. Identifying the role of woman in bringing up children on respecting freedom.

5. Providing a proposal of woman's role in achieving intellectual safety in the Saudi community.

\subsection{Significance}

1. Shedding light on the role of woman in achieving intellectual safety for the youth in the Saudi community as the driving force of the family.

2. Intellectual safety is a prerequisite requirement for each nation and is mainly formed in the family.

3. Highlighting the role of woman in activating dialogue culture, implanting positive values among youth, and reinforcing human self in the family. 
4. Providing a proposal of woman's role in achieving intellectual safety in the Saudi community that can be used by family institutions as a mechanism of enlightenment in achieving intellectual safety.

\subsection{Definition of Terms}

The role: The role is a set of socially defined adjectives and expectations that are related to a certain status (Ameen, 2012, p.7). In the study, it is the set of actions, practices, guidelines, and pieces of advice that should be conducted by the woman towards her family members to achieve intellectual safety.

Intellectual safety: Protecting the human's mind, thinking, inventions, knowledge, products, perspectives, and freedom of opinion from any influence, either by the person or by others (Eldeaj, 1985, p. 104). In the study, it is the sound thinking of family members and understanding and making their minds free from wrong beliefs and ideas causing deviation and violating modesty in understanding and thinking, whether in religious or secular issues.

\section{Procedures}

The chapter covers an illustration of the study's type, method, population, description of performance, checking validity and reliability, and statistical methods.

\section{Method and sample}

The study is a descriptive and analytical one that is interested in describing a phenomenon (the role of woman in achieving intellectual safety in the Saudi community) and revealing the relationship between the role of woman in achieving intellectual safety and the related variables.

The study utilized social survey method to a randomly selected sample of (1300) female students of Saudi universities in Riyadh (Princess Nourah bint Abddulrahman University, King Saud University, and Imam Muhammad ibn Saud Islamic University) for the first semester of the academic year 2016/2017.

\section{Tools of the study: The study adopted the questionnaire as a key tool for collecting data.}

\section{Validity and reliability of the tool}

\section{a. Face Validity}

To identify the validity of the study's tool, it was displayed to (35) reviewers of Ph.D. holders, with (5) years of experience, and those specialized in social work and related sciences. According to their opinions, the questionnaire was prepared in its final form.

\section{b. Content Validity}

Pearson Correlation Coefficient was estimated to define the content validity of the questionnaire, where correlation coefficient of each item was estimated to the total degree of the domain, as follows:

Table 1. Pearson Correlation Coefficient of the first domain's items to the total score

\begin{tabular}{|c|c|c|c|}
\hline No. & $\begin{array}{c}\text { Correlation } \\
\text { coefficient }\end{array}$ & No. & $\begin{array}{c}\text { Correlation } \\
\text { coefficient }\end{array}$ \\
\hline 1 & $0.735^{* *}$ & 6 & $0.751^{* *}$ \\
\hline 2 & $0.669^{* *}$ & 7 & $0.785^{* *}$ \\
\hline 3 & $0.709^{* *}$ & 8 & $0.731^{* *}$ \\
\hline 4 & $0.616^{* *}$ & 9 & $0.769^{* *}$ \\
\hline 5 & $0.762^{* *}$ & - & - \\
\hline
\end{tabular}

** Significant at the level of 0.01 and less

Table 2. Pearson Correlation Coefficient of the second domain's items to the total score

\begin{tabular}{|c|c|c|c|}
\hline No. & $\begin{array}{c}\text { Correlation } \\
\text { coefficient }\end{array}$ & No. & $\begin{array}{c}\text { Correlation } \\
\text { coefficient }\end{array}$ \\
\hline 1 & $0.542^{* *}$ & 10 & $0.617^{* *}$ \\
\hline 2 & $0.575^{* *}$ & 11 & $0.635^{* *}$ \\
\hline 3 & $0.612 * *$ & 12 & $0.589^{* *}$ \\
\hline 4 & $0.558 * *$ & 13 & $0.687^{* *}$ \\
\hline 5 & $0.585^{* *}$ & 14 & $0.640^{* *}$ \\
\hline 6 & $0.650 * *$ & 15 & $0.672^{* *}$ \\
\hline 7 & $0.578 * *$ & 16 & $0.701^{* *}$ \\
\hline 8 & $0.537 * *$ & 17 & $0.657^{* *}$ \\
\hline 9 & $0.578 * *$ & - & - \\
\hline
\end{tabular}

** Significant at the level of 0.01 and less

Table 3. Pearson Correlation Coefficient of the third domain's items to the total score

\begin{tabular}{|c|c|c|c|}
\hline No. & $\begin{array}{c}\text { Correlation } \\
\text { coefficient }\end{array}$ & No. & $\begin{array}{c}\text { Correlation } \\
\text { coefficient }\end{array}$ \\
\hline 1 & $0.638^{* *}$ & 8 & $0.714^{* *}$ \\
\hline 2 & $0.701^{* *}$ & 9 & $0.744^{* *}$ \\
\hline 3 & $0.764 * *$ & 10 & $0.705^{* *}$ \\
\hline 4 & $0.660^{* *}$ & 11 & $0.786^{* *}$ \\
\hline 5 & $0.676^{* *}$ & 12 & $0.725^{* *}$ \\
\hline 6 & $0.736^{* *}$ & 13 & $0.717^{* *}$ \\
\hline 7 & $0.735^{* *}$ & - & - \\
\hline
\end{tabular}

** Significant at the level of 0.01 and less

Table 4. Pearson Correlation Coefficient of the fourth domain's items to the total score

\begin{tabular}{|c|c|c|c|}
\hline No. & $\begin{array}{c}\text { Correlation } \\
\text { coefficient }\end{array}$ & No. & $\begin{array}{c}\text { Correlation } \\
\text { coefficient }\end{array}$ \\
\hline 1 & $0.708 * *$ & 6 & $0.757 * *$ \\
\hline 2 & $0.677 * *$ & 7 & $0.777 * *$ \\
\hline 3 & $0.733 * *$ & 8 & $0.763 * *$ \\
\hline 4 & $0.693 * *$ & 9 & $0.752^{* *}$ \\
\hline 5 & $0.726 * *$ & - & - \\
\hline
\end{tabular}

** Significant at the level of 0.01 and less

Tables 1 and 4 show that the values of correlation 
coefficient of each item with its domain are positive and statistically significant at the level of (0.01) and less, indicating their content validity with the domain.

\section{Reliability of the tool}

To estimate the reliability of the tool, Cronbach's Alpha $(\alpha)$ was estimated. Table 5 illustrates the reliability coefficients of the tool.

Table 5. Cronbach's Alpha $(\alpha)$ to measure the reliability of the tool

\begin{tabular}{|l|c|c|}
\hline \multicolumn{1}{|c|}{ Domains } & $\begin{array}{c}\text { No. of } \\
\text { items }\end{array}$ & $\begin{array}{c}\text { Reliability } \\
\text { of the } \\
\text { domain }\end{array}$ \\
\hline $\begin{array}{l}\text { The role of woman in bringing up children } \\
\text { according to the culture and mechanism of } \\
\text { family dialogue }\end{array}$ & 9 & 0.8863 \\
\hline $\begin{array}{l}\text { The role of woman in implanting moderate } \\
\text { Islamic values in children }\end{array}$ & 17 & 0.8951 \\
\hline $\begin{array}{l}\text { The role of woman in reinforcing, respecting, } \\
\text { and improving the self and maintaining } \\
\text { cultural distinctiveness. }\end{array}$ & 13 & 0.9201 \\
\hline $\begin{array}{l}\text { The role of woman in bringing up children on } \\
\text { respecting freedom of others. }\end{array}$ & 9 & 0.8907 \\
\hline General reliability & 48 & 0.9570 \\
\hline
\end{tabular}

Table 5 illustrates that general reliability coefficient is high, scoring $(0.9570)$. This indicates that the questionnaire has high-reliability degree that can be used in the field application.

\section{Statistical processing methods}

To achieve objectives and analyze data, various statistical methods were adopted using SPSS (Statistical Package for Social Sciences)

Then, the following methods were calculated:

1. Frequencies and percentages to define the personal and occupational features of the participants and their responses to the items of the domain.

2. The weighted mean was estimated to define the increase or decrease of responses to the items of each key variable, as follows:
- $\quad$ From (1) to (1.67); Not achieved

- $\quad$ From (1.68) to (2.34); Somewhat achieved

- $\quad$ From (2.35) to (3.00); Achieved

3. The arithmetic mean was estimated to define the increase or decrease of responses to the domains (means of the items). They are ranged from the highest.

4. Standard deviation was estimated to define the deviations of the items and each domain.

\section{Fields of the study}

The population of the study comprised female students of the Saudi universities in Riyadh in Princess Nourah bint Abddulrahman University, King Saud University, and Imam Muhammad ibn Saud Islamic University for the first semester of the academic year 2016/2017.

\section{A. Description of the items}

Table 6. Distribution of the items according to the university's variable

\begin{tabular}{|c|c|c|}
\hline University & Frequency & Percentage \\
\hline $\begin{array}{c}\text { Princess Nourah bint Abddulrahman } \\
\text { University }\end{array}$ & 600 & 46.2 \\
\hline King Saud University & 350 & 26.9 \\
\hline $\begin{array}{c}\text { Al-Imam Muhammad Ibn Saud Islamic } \\
\text { University }\end{array}$ & 350 & 26.9 \\
\hline Total & 1300 & $100 \%$ \\
\hline
\end{tabular}

\section{Discussion}

1st question: "What is the role of woman in bringing up children according to the culture and mechanism of family dialogue?"

To identify the role of woman in bringing up children according to the culture and mechanism of family dialogue, frequencies, percentages, arithmetic means, standard deviations, and ranks of the responses to the items of the domain. Results are shown in

Table 7. Responses of the participants to the items of "the role of woman in bringing up children according to the culture and mechanism of family dialogue" domain in a descending order according to the agreement

\begin{tabular}{|c|c|c|c|c|c|c|c|c|c|}
\hline \multirow[b]{2}{*}{ No. } & \multirow{2}{*}{\multicolumn{2}{|c|}{ Item }} & Frequency & \multicolumn{3}{|c|}{ Agreement degree } & \multirow{2}{*}{$\begin{array}{c}\text { Arithmetic } \\
\text { mean }\end{array}$} & \multirow{2}{*}{$\begin{array}{l}\text { Standard } \\
\text { deviation }\end{array}$} & \multirow[b]{2}{*}{ Rank } \\
\hline & & & Percentage & Achieved & $\begin{array}{c}\text { Somewhat } \\
\text { achieved }\end{array}$ & $\begin{array}{c}\text { Not } \\
\text { achieved }\end{array}$ & & & \\
\hline \multirow{8}{*}{1} & \multirow{8}{*}{$\begin{array}{l}\text { The mother educates } \\
\text { the children. }\end{array}$} & \multirow{2}{*}{$\begin{array}{c}\text { Princess } \\
\text { Nourah bint } \\
\text { Abddulrahman } \\
\text { University }\end{array}$} & Freq. & 404 & 181 & 15 & \multirow[b]{2}{*}{2.65} & \multirow[b]{2}{*}{0.528} & \multirow{8}{*}{1} \\
\hline & & & $\%$ & 67.3 & 30.2 & 2.5 & & & \\
\hline & & \multirow{2}{*}{$\begin{array}{l}\text { King Saud } \\
\text { University }\end{array}$} & Freq. & 212 & 127 & 11 & \multirow{2}{*}{2.57} & \multirow{2}{*}{0.555} & \\
\hline & & & $\%$ & 60.6 & 36.3 & 3.1 & & & \\
\hline & & \multirow{2}{*}{$\begin{array}{c}\text { Imam } \\
\text { Muhammad } \\
\text { ibn Saud } \\
\text { Islamic } \\
\text { University }\end{array}$} & Freq. & 246 & 94 & 10 & \multirow[b]{2}{*}{2.67} & \multirow[b]{2}{*}{0.527} & \\
\hline & & & $\%$ & 70.3 & 26.9 & 2.9 & & & \\
\hline & & \multirow{2}{*}{ Total } & Freq. & 862 & 402 & 36 & \multirow{2}{*}{2.64} & \multirow{2}{*}{0.536} & \\
\hline & & & $\%$ & 66.3 & 30.9 & 2.8 & & & \\
\hline
\end{tabular}




\begin{tabular}{|c|c|c|c|c|c|c|c|c|c|}
\hline \multirow[b]{2}{*}{ No. } & \multirow{2}{*}{\multicolumn{2}{|c|}{ Item }} & Frequency & \multicolumn{3}{|c|}{ Agreement degree } & \multirow{2}{*}{$\begin{array}{l}\text { Arithmetic } \\
\text { mean }\end{array}$} & \multirow{2}{*}{$\begin{array}{l}\text { Standard } \\
\text { deviation }\end{array}$} & \multirow[b]{2}{*}{ Rank } \\
\hline & & & Percentage & Achieved & $\begin{array}{c}\text { Somewhat } \\
\text { achieved }\end{array}$ & $\begin{array}{c}\text { Not } \\
\text { achieved }\end{array}$ & & & \\
\hline \multirow{8}{*}{2} & \multirow{8}{*}{$\begin{array}{c}\text { Introducing the } \\
\text { effects of } \\
\text { intellectual } \\
\text { deviation to children }\end{array}$} & \multirow{2}{*}{$\begin{array}{c}\text { Princess } \\
\text { Nourah bint } \\
\text { Abddulrahman } \\
\text { University }\end{array}$} & Freq. & 306 & 256 & 38 & \multirow[b]{2}{*}{2.45} & \multirow[b]{2}{*}{0.612} & \multirow{8}{*}{9} \\
\hline & & & $\%$ & 51.0 & 42.7 & 6.3 & & & \\
\hline & & \multirow{2}{*}{$\begin{array}{l}\text { King Saud } \\
\text { University }\end{array}$} & Freq. & 158 & 178 & 14 & \multirow{2}{*}{2.41} & \multirow{2}{*}{0.568} & \\
\hline & & & $\%$ & 45.1 & 50.9 & 4.0 & & & \\
\hline & & \multirow{2}{*}{$\begin{array}{c}\text { Imam } \\
\text { Muhammad } \\
\text { ibn Saud } \\
\text { Islamic } \\
\text { University }\end{array}$} & Freq. & 184 & 138 & 28 & \multirow[b]{2}{*}{2.45} & \multirow[b]{2}{*}{0.639} & \\
\hline & & & $\%$ & 52.6 & 39.4 & 8.0 & & & \\
\hline & & \multirow{2}{*}{ Total } & Freq. & 648 & 572 & 80 & \multirow{2}{*}{2.44} & \multirow{2}{*}{0.608} & \\
\hline & & & $\%$ & 49.8 & 44.0 & 6.2 & & & \\
\hline \multirow{8}{*}{3} & \multirow{8}{*}{$\begin{array}{l}\text { Introducing the } \\
\text { effects of behavioral } \\
\text { deviation to children }\end{array}$} & \multirow{2}{*}{$\begin{array}{c}\text { Princess } \\
\text { Nourah bint } \\
\text { Abddulrahman } \\
\text { University } \\
\end{array}$} & Freq. & 369 & 197 & 34 & \multirow[b]{2}{*}{2.56} & \multirow[b]{2}{*}{0.600} & \\
\hline & & & $\%$ & 61.5 & 32.8 & 5.7 & & & \\
\hline & & King Saud & Freq. & 185 & 146 & 19 & 247 & 0.599 & \\
\hline & & University & $\%$ & 52.9 & 41.7 & 5.4 & 2.47 & 0.599 & \\
\hline & & Imam & Freq. & 228 & 99 & 23 & & & 7 \\
\hline & & $\begin{array}{l}\text { Muhammad } \\
\text { ibn Saud } \\
\text { Islamic } \\
\text { University } \\
\end{array}$ & $\%$ & 65.1 & 28.3 & 6.6 & 2.59 & 0.612 & \\
\hline & & & Freq. & 782 & 442 & 76 & & & \\
\hline & & Total & $\%$ & 60.2 & 34.0 & 5.8 & 2.54 & 0.604 & \\
\hline & & Princess & Freq. & 386 & 177 & 37 & & & \\
\hline & & $\begin{array}{l}\text { Nourah bint } \\
\text { Abddulrahman } \\
\text { University }\end{array}$ & $\%$ & 64.3 & 29.5 & 6.2 & 2.58 & 0.606 & \\
\hline & & King Saud & Freq. & 208 & 117 & 25 & 2.52 & 0.627 & \\
\hline & rules regulating the & University & $\%$ & 59.4 & 33.4 & 7.1 & 2.52 & 0.021 & \\
\hline 4 & relationship between & Imam & Freq. & 234 & 94 & 22 & & & 5 \\
\hline & ruled. & $\begin{array}{l}\text { Munammad } \\
\text { ibn Saud } \\
\text { Islamic } \\
\text { University } \\
\end{array}$ & $\%$ & 66.9 & 26.9 & 6.3 & 2.61 & 0.605 & \\
\hline & & Total & Freq. & 828 & 388 & 84 & 257 & 0612 & \\
\hline & & Total & $\%$ & 63.7 & 29.8 & 6.5 & 2.57 & 0.612 & \\
\hline & & Princess & Freq. & 374 & 180 & 46 & & & \\
\hline & & $\begin{array}{l}\text { Nourah bint } \\
\text { Abddulrahman } \\
\text { University }\end{array}$ & $\%$ & 62.3 & 30.0 & 7.7 & 2.55 & 0.634 & \\
\hline & & King Saud & Freq. & 189 & 124 & 37 & & & \\
\hline & The mother is & University & $\%$ & 54.0 & 35.4 & 10.6 & 2.43 & 0.677 & \\
\hline 5 & $\begin{array}{l}\text { interested in the } \\
\text { family dialogue }\end{array}$ & Imam & Freq. & 229 & 99 & 22 & & & 8 \\
\hline & among children. & $\begin{array}{l}\text { ibn Saud } \\
\text { Islamic } \\
\text { University } \\
\end{array}$ & $\%$ & 65.4 & 28.3 & 6.3 & 2.59 & 0.607 & \\
\hline & & & Freq. & 792 & 403 & 105 & & & \\
\hline & & Total & $\%$ & 60.9 & 31.0 & 8.1 & 2.53 & 0.641 & \\
\hline & $\begin{array}{l}\text { The mother is } \\
\text { The }\end{array}$ & Princess & Freq. & 396 & 165 & 39 & & & \\
\hline 6 & $\begin{array}{l}\text { interested in solving } \\
\text { problems and } \\
\text { disagreements }\end{array}$ & $\begin{array}{l}\text { Nourah bint } \\
\text { Abddulrahman } \\
\text { University }\end{array}$ & $\%$ & 66.0 & 27.5 & 6.5 & 2.60 & 0.610 & 4 \\
\hline
\end{tabular}




\begin{tabular}{|c|c|c|c|c|c|c|c|c|c|}
\hline \multirow{8}{*}{ No. } & \multirow{2}{*}{\multicolumn{2}{|c|}{ Item }} & Frequency & \multicolumn{3}{|c|}{ Agreement degree } & \multirow{2}{*}{$\begin{array}{l}\text { Arithmetic } \\
\text { mean }\end{array}$} & \multirow{2}{*}{$\begin{array}{l}\text { Standard } \\
\text { deviation }\end{array}$} & \multirow{8}{*}{ Rank } \\
\hline & & & Percentage & Achieved & $\begin{array}{l}\text { Somewhat } \\
\text { achieved }\end{array}$ & $\begin{array}{c}\text { Not } \\
\text { achieved }\end{array}$ & & & \\
\hline & \multirow[t]{6}{*}{ among children. } & \multirow{2}{*}{$\begin{array}{l}\text { King Saud } \\
\text { University }\end{array}$} & Freq. & 193 & 127 & 30 & \multirow{2}{*}{2.47} & \multirow{2}{*}{0.649} & \\
\hline & & & $\%$ & 55.1 & 36.3 & 8.6 & & & \\
\hline & & \multirow{2}{*}{$\begin{array}{c}\text { Imam } \\
\text { Muhammad } \\
\text { ibn Saud } \\
\text { Islamic } \\
\text { University } \\
\end{array}$} & Freq. & 254 & 76 & 20 & \multirow[b]{2}{*}{2.67} & \multirow[b]{2}{*}{0.580} & \\
\hline & & & $\%$ & 72.6 & 21.7 & 5.7 & & & \\
\hline & & \multirow{2}{*}{ Total } & Freq. & 843 & 368 & 89 & \multirow{2}{*}{2.58} & \multirow{2}{*}{0.617} & \\
\hline & & & $\%$ & 64.8 & 28.3 & 6.8 & & & \\
\hline \multirow{8}{*}{7} & \multirow{8}{*}{$\begin{array}{l}\text { The mother corrects } \\
\text { wrong concepts } \\
\text { among children. }\end{array}$} & \multirow{2}{*}{$\begin{array}{c}\text { Princess } \\
\text { Nourah bint } \\
\text { Abddulrahman } \\
\text { University }\end{array}$} & Freq. & 412 & 162 & 26 & \multirow[b]{2}{*}{2.64} & \multirow[b]{2}{*}{0.563} & \multirow{8}{*}{3} \\
\hline & & & $\%$ & 68.7 & 27.0 & 4.3 & & & \\
\hline & & King Saud & Freq. & 206 & 123 & 21 & 253 & 0608 & \\
\hline & & University & $\%$ & 58.9 & 35.1 & 6.0 & 2.03 & 0.000 & \\
\hline & & Imam & Freq. & 260 & 80 & 10 & & & \\
\hline & & $\begin{array}{l}\text { ibn Saud } \\
\text { Islamic } \\
\text { University }\end{array}$ & $\%$ & 74.3 & 22.9 & 2.9 & 2.71 & 0.512 & \\
\hline & & \multirow{2}{*}{ Total } & Freq. & 878 & 365 & 57 & \multirow{2}{*}{2.63} & \multirow{2}{*}{0.566} & \\
\hline & & & $\%$ & 67.5 & 28.1 & 4.4 & & & \\
\hline \multirow{8}{*}{8} & \multirow{8}{*}{$\begin{array}{l}\text { Introducing others' } \\
\text { rights to children. }\end{array}$} & \multirow{2}{*}{$\begin{array}{c}\text { Princess } \\
\text { Nourah bint } \\
\text { Abddulrahman } \\
\text { University }\end{array}$} & Freq. & 380 & 183 & 37 & & & \\
\hline & & & $\%$ & 63.3 & 30.5 & 6.2 & 2.57 & 0.607 & \\
\hline & & King Saud & Freq. & 190 & 136 & 24 & 17 & 000 & \\
\hline & & University & $\%$ & 54.3 & 38.9 & 6.9 & 2.71 & 0.025 & \\
\hline & & Imam & Freq. & 245 & 85 & 20 & & & 6 \\
\hline & & $\begin{array}{l}\text { ibn Saud } \\
\text { Islamic } \\
\text { University } \\
\end{array}$ & $\%$ & 70.0 & 24.3 & 5.7 & 2.64 & 0.587 & \\
\hline & & Total & Freq. & 815 & 404 & 81 & 256 & 0600 & \\
\hline & & Torat & $\%$ & 62.7 & 31.1 & 6.2 & 2.50 & 0.00 & \\
\hline & & Princess & Freq. & 413 & 164 & 23 & & & \\
\hline & & $\begin{array}{c}\text { Abddulrahman } \\
\text { University }\end{array}$ & $\%$ & 68.8 & 27.3 & 3.8 & 2.65 & 0.552 & \\
\hline & & King Saud & Freq. & 205 & 126 & 19 & 253 & 590 & \\
\hline & The mother uses & University & $\%$ & 58.6 & 36.0 & 5.4 & 2.00 & צ & \\
\hline 9 & implement morals & Imam & Freq. & 263 & 76 & 11 & & & 2 \\
\hline & and ethics. & $\begin{array}{l}\text { ibn Saud } \\
\text { Islamic } \\
\text { University } \\
\end{array}$ & $\%$ & 75.1 & 21.7 & 3.1 & 2.72 & 0.515 & \\
\hline & & Total & Freq. & 881 & 366 & 53 & 264 & 0559 & \\
\hline & & I0tal & $\%$ & 67.8 & 28.2 & 4.1 & 2.04 & 0.539 & \\
\hline & & GeI & eral mean & & & & 2.57 & & \\
\hline
\end{tabular}


Table 7 illustrates that the agreement of the participants to $"$ the role of woman in bringing up children according to the culture and mechanism of family dialogue" achieved an arithmetic mean 2.57 out of 3.00 ), which was ranked third on the triple scale (from 2.35 out of 3.00), indicating its achievement.

Accordingly, there is consistency of their agreement. They agreed on nine roles, the most significant of which are $(1,9$, 7,6 , and 4 ) in a descending order, as follows:

1. Item (1) "The mother educates children" was ranked first, with an arithmetic mean 2.64 out of 4 . The female students of Imam Muhammad ibn Saud Islamic University were ranked the highest with an arithmetic mean 2.67 out of 4 ; followed by those of Princess Nourah bint Abddulrahman University, with an arithmetic mean 2.65 out of 4; and those of King Saud University, with an arithmetic mean 2.57 out of 4 .

2. Item (9) "the mother uses dialogue to implement morals and ethics" was ranked second, with an arithmetic mean 2.64 out of 4. The female students of Imam Muhammad ibn Saud Islamic University were ranked the highest with an arithmetic mean 2.72 out of 4; followed by those of Princess Nourah bint Abddulrahman University, with an arithmetic mean 2.65 out of 4 ; and those of King Saud University, with an arithmetic mean 2.53 out of 4 .

3. Item (7) "the mother corrects wrong concepts among children" was ranked third, with an arithmetic mean 2.63 out of 4. The female students of Imam Muhammad ibn Saud Islamic University were ranked the highest with an arithmetic mean 2.71 out of 4; followed by those of Princess Nourah bint Abddulrahman University, with an arithmetic mean 2.64 out of 4; and those of King Saud University, with an arithmetic mean 2.53 out of 4 .

4. Item (6) "the mother is interested in solving problems and disagreements among children" was ranked fourth, with an arithmetic mean 2.58 out of 4 . The female students of Imam Muhammad ibn Saud Islamic University were ranked the highest with an arithmetic mean 2.67 out of 4 ; followed by those of Princess Nourah bint Abddulrahman University, with an arithmetic mean 2.60 out of 4, and those of King Saud University, with an arithmetic mean 2.47 out of 4 .
Item (4) "introducing the legal rules regulating the relationship between the ruler and the ruled" was ranked fifth, with an arithmetic mean 2.57 out of 4 . The female students of Imam Muhammad ibn Saud Islamic University were ranked the highest with an arithmetic mean 2.61 out of 4 ; followed by those of Princess Nourah bint Abddulrahman University, with an arithmetic mean 2.58 out of 4 ; and those of King Saud University, with an arithmetic mean 2.52 out of 4 .

Results attest that the most significant roles of woman in bringing up children according to the culture and mechanism of family dialogue are that she "educates children" because she is keen on bringing them up in a way they adopt with community by acquiring the permanent community culture. This is consistent with the related literature.

It is consistent with Eljahny (2004) illustrating that youth personality is formed by the conduct practices of the family.

This affirms the role of woman in activating the culture and mechanism of family dialogue and her impact on brining-up children and forming their personality. This is reflected in their ideas, attitudes, and treatment with others.

In addition, it is consistent with the nature of the dominant relationship with adolescents and parents. A family faces a dilemma because of the ignorance of many parents of their responsibilities towards protecting their children from deviations, absence of good family dialogue causing various behavioral disorders, particularly intellectual deviation.

Arab and foreign literature is consistent regarding these results, e.g. Katsos (2003) that affirms that more attention should be paid to human relations, focusing on values and personality building, high awareness by perceiving diversity and implanting tolerance, and increasing the procedures and scales of security.

The results are also consistent with the related theoretical literature regarding the role of woman in forming sons' personality and affecting them to accomplish a comprehensive social education as the family still maintains its positive role in bringing him up in a time he lacks some personality and social bases (Elsaleh, 2004). This can be achieved through various family educational responsibilities of the family and woman, the most significant of which are: Educating children on creative dialogue, respecting opinions, and disseminating dialogue culture in the family. Purposeful communication is needed with the institutions that children deal with, e.g. school, university, and security authorities. 
Table 8. Responses of the participants to the items of "the role of woman in implanting moderate Islamic values in children" domain in a descending order according to the agreement

\begin{tabular}{|c|c|c|c|c|c|c|c|c|c|}
\hline \multirow[b]{2}{*}{ No. } & \multirow{2}{*}{\multicolumn{2}{|c|}{ Item }} & Frequency & \multicolumn{3}{|c|}{ Agreement degree } & \multirow{2}{*}{$\begin{array}{c}\text { Arithmetic } \\
\text { mean }\end{array}$} & \multirow{2}{*}{$\begin{array}{l}\text { Standard } \\
\text { deviation }\end{array}$} & \multirow[b]{2}{*}{ Rank } \\
\hline & & & Percentage & Agree & Somewhat & Disagree & & & \\
\hline \multirow{7}{*}{1} & \multirow{7}{*}{$\begin{array}{l}\text { Guiding children } \\
\text { not to rush into } \\
\text { making judgments. }\end{array}$} & \multirow{2}{*}{$\begin{array}{c}\text { Princess } \\
\text { Nourah bint } \\
\text { Abddulrahman } \\
\text { University }\end{array}$} & Freq. & 437 & 153 & 10 & \multirow[b]{2}{*}{2.71} & \multirow[b]{2}{*}{0.489} & \multirow{7}{*}{13} \\
\hline & & & $\%$ & 72.8 & 25.5 & 1.7 & & & \\
\hline & & $\begin{array}{l}\text { King Saud } \\
\text { University }\end{array}$ & $\%$ & 69.4 & 28.3 & 2.3 & 2.67 & 0.517 & \\
\hline & & \multirow{2}{*}{$\begin{array}{c}\text { Imam } \\
\text { Muhammad } \\
\text { ibn Saud } \\
\text { Islamic } \\
\text { University }\end{array}$} & Freq. & 262 & 83 & 5 & \multirow[b]{2}{*}{2.73} & \multirow[b]{2}{*}{0.474} & \\
\hline & & & $\%$ & 74.9 & 23.7 & 1.4 & & & \\
\hline & & \multirow{2}{*}{ Total } & Freq. & 942 & 335 & 23 & \multirow{2}{*}{2.71} & \multirow{2}{*}{0.493} & \\
\hline & & & $\%$ & 72.5 & 25.8 & 1.8 & & & \\
\hline \multirow{8}{*}{2} & \multirow{8}{*}{$\begin{array}{l}\text { Warning children } \\
\text { not to follow } \\
\text { mistrust }\end{array}$} & \multirow{2}{*}{$\begin{array}{c}\text { Princess } \\
\text { Nourah bint } \\
\text { Abddulrahman } \\
\text { University } \\
\end{array}$} & Freq. & 455 & 136 & 9 & \multirow[b]{2}{*}{2.74} & \multirow[b]{2}{*}{0.470} & \\
\hline & & & $\%$ & 75.8 & 22.7 & 1.5 & & & \\
\hline & & King Saud & Freq. & 260 & 86 & 4 & 273 & 0460 & \\
\hline & & University & $\%$ & 74.3 & 24.6 & 1.1 & 2.10 & 0.70 & \\
\hline & & Imam & Freq. & 268 & 75 & 7 & & & 7 \\
\hline & & $\begin{array}{l}\text { ibn Saud } \\
\text { Islamic } \\
\text { University }\end{array}$ & $\%$ & 76.6 & 21.4 & 2.0 & 2.75 & 0.480 & \\
\hline & & Totol & Freq. & 983 & 297 & 20 & 274 & 0477 & \\
\hline & & Forat & $\%$ & 75.6 & 22.8 & 1.5 & 2.17 & 0.712 & \\
\hline & & $\begin{array}{c}\text { Abddulrahman } \\
\text { University }\end{array}$ & $\%$ & 81.5 & 17.7 & 0.8 & 2.81 & 0.416 & \\
\hline & & King Saud & Freq. & 278 & 69 & 3 & 270 & 0431 & \\
\hline & Illustrating the & University & $\%$ & 79.4 & 19.7 & 0.9 & 2.19 & 0.431 & \\
\hline 3 & self-aggression and & Imam & Freq. & 290 & 56 & 4 & & & 3 \\
\hline & avoiding injustice & $\begin{array}{l}\text { ibn Saud } \\
\text { Islamic } \\
\text { University }\end{array}$ & $\%$ & 82.9 & 16.0 & 1.1 & 2.82 & 0.416 & \\
\hline & & Total & Freq. & 1057 & 231 & 12 & 280 & 040 & \\
\hline & & 10 tal & $\%$ & 81.3 & 17.8 & 0.9 & 2.00 & 0.420 & \\
\hline & & Princess & Freq. & 472 & 122 & 6 & & & \\
\hline & & $\begin{array}{l}\text { Abourah bint } \\
\text { University }\end{array}$ & $\%$ & 78.7 & 20.3 & 1.0 & 2.78 & 0.440 & \\
\hline & & King Saud & Freq. & 261 & 85 & 4 & 273 & 0468 & \\
\hline & & University & $\%$ & 74.6 & 24.3 & 1.1 & 2.13 & 0.400 & \\
\hline 4 & good friends & Imam & Freq. & 286 & 62 & 2 & & & 5 \\
\hline & & $\begin{array}{l}\text { ibn Saud } \\
\text { Islamic } \\
\text { University }\end{array}$ & $\%$ & 81.7 & 17.7 & 0.6 & 2.81 & 0.406 & \\
\hline & & Total & Freq. & 1019 & 269 & 12 & 277 & 0440 & \\
\hline & & Hotal & $\%$ & 78.4 & 20.7 & 0.9 & 2.17 & 0.440 & \\
\hline & & Princess & Freq. & 511 & 86 & 3 & & & \\
\hline 5 & $\begin{array}{l}\text { Motivating } \\
\text { children to adhere } \\
\text { to social morals. }\end{array}$ & $\begin{array}{l}\text { Nourah bint } \\
\text { Abddulrahman } \\
\text { University }\end{array}$ & $\%$ & 85.2 & 14.3 & 0.5 & 2.85 & 0.374 & 2 \\
\hline
\end{tabular}




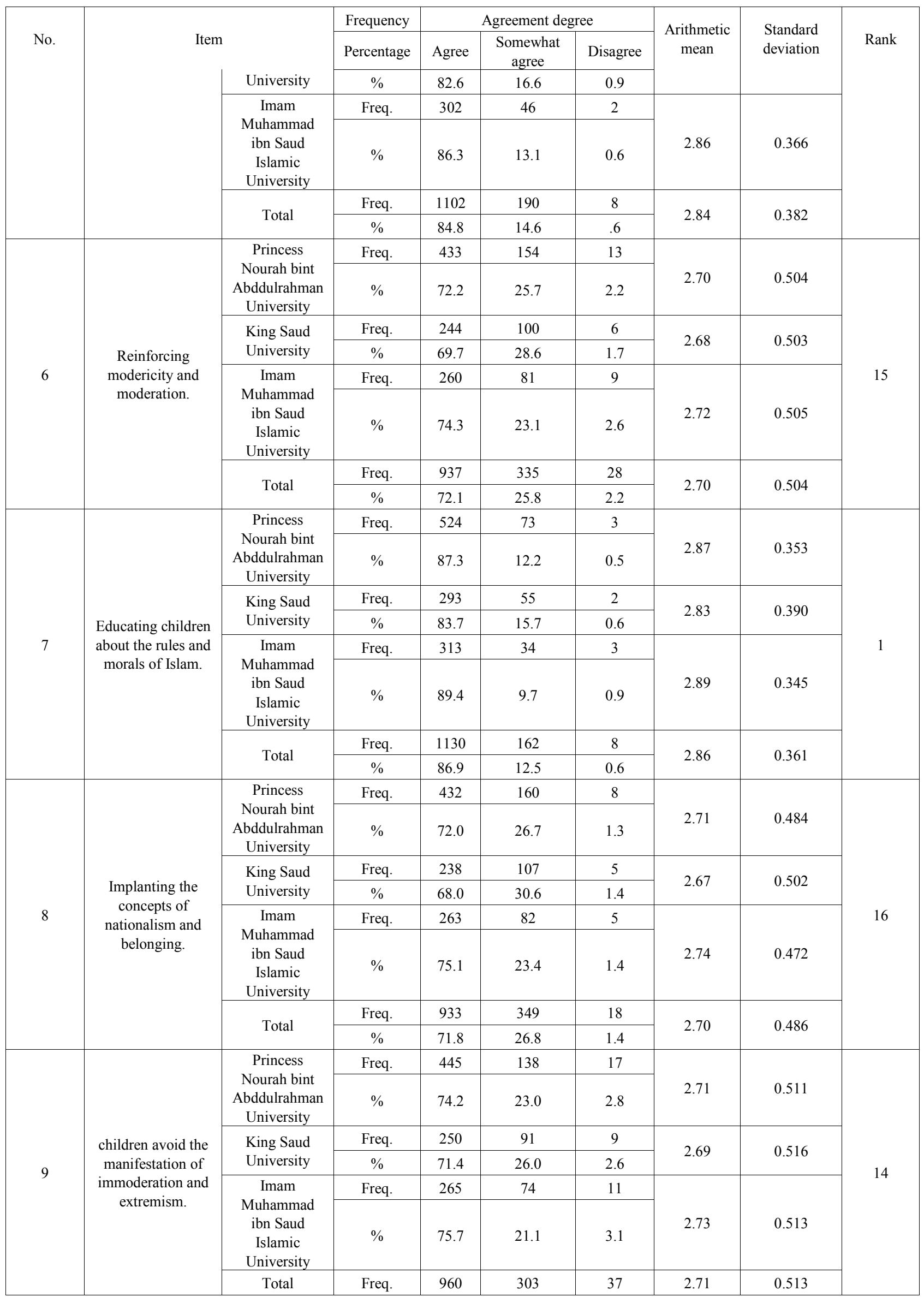




\begin{tabular}{|c|c|c|c|c|c|c|c|c|c|}
\hline \multirow{3}{*}{ No. } & \multirow{3}{*}{ Item } & & \multirow{3}{*}{$\begin{array}{c}\text { Frequency } \\
\text { Percentage } \\
\%\end{array}$} & \multicolumn{3}{|c|}{ Agreement degree } & \multirow{3}{*}{$\begin{array}{l}\text { Arithmetic } \\
\text { mean }\end{array}$} & \multirow{3}{*}{$\begin{array}{l}\text { Standard } \\
\text { deviation }\end{array}$} & \multirow{3}{*}{ Rank } \\
\hline & & & & \multirow{2}{*}{\multicolumn{2}{|c|}{\begin{tabular}{|c|}
$\begin{array}{c}\text { Somewhat } \\
\text { agree }\end{array}$ \\
23.3 \\
\end{tabular}}} & \multirow{2}{*}{$\begin{array}{c}\text { Disagree } \\
2.8 \\
\end{array}$} & & & \\
\hline & & & & & & & & & \\
\hline \multirow{8}{*}{10} & \multirow{8}{*}{$\begin{array}{l}\text { Children avoid the } \\
\text { manifestation of } \\
\text { behavioral } \\
\text { deviation. }\end{array}$} & \multirow{2}{*}{$\begin{array}{c}\text { Princess } \\
\text { Nourah bint } \\
\text { Abddulrahman } \\
\text { University }\end{array}$} & Freq. & 481 & 109 & 10 & \multirow[b]{2}{*}{2.78} & \multirow[b]{2}{*}{0.450} & \multirow{8}{*}{4} \\
\hline & & & $\%$ & 80.2 & 18.2 & 1.7 & & & \\
\hline & & \multirow{2}{*}{$\begin{array}{l}\text { King Saud } \\
\text { University }\end{array}$} & Freq. & 269 & 75 & 6 & \multirow{2}{*}{2.75} & \multirow{2}{*}{0.471} & \\
\hline & & & $\%$ & 76.9 & 21.4 & 1.7 & & & \\
\hline & & \multirow{2}{*}{$\begin{array}{c}\text { Imam } \\
\text { Muhammad } \\
\text { ibn Saud } \\
\text { Islamic } \\
\text { University }\end{array}$} & Freq. & 284 & 61 & 5 & & & \\
\hline & & & $\%$ & 81.1 & 17.4 & 1.4 & 2.80 & 0.437 & \\
\hline & & \multirow{2}{*}{ Total } & Freq. & 1034 & 245 & 21 & \multirow{2}{*}{2.78} & \multirow{2}{*}{0.452} & \\
\hline & & & $\%$ & 79.5 & 18.8 & 1.6 & & & \\
\hline \multirow{8}{*}{11} & \multirow{8}{*}{$\begin{array}{l}\text { Avoiding verbal } \\
\text { violence with sons. }\end{array}$} & Princess & Freq. & 443 & 141 & 16 & & & \\
\hline & & $\begin{array}{l}\text { Nourah bint } \\
\text { Abddulrahman } \\
\text { University }\end{array}$ & $\%$ & 73.8 & 23.5 & 2.7 & 2.71 & 0.509 & \\
\hline & & King Saud & Freq. & 238 & 102 & 10 & & & \\
\hline & & University & $\%$ & 68.0 & 29.1 & 2.9 & 2.65 & 0.534 & \\
\hline & & Imam & Freq. & 269 & 69 & 12 & & & 17 \\
\hline & & $\begin{array}{l}\text { ibn Saud } \\
\text { Islamic } \\
\text { University } \\
\end{array}$ & $\%$ & 76.9 & 19.7 & 3.4 & 2.73 & 0.514 & \\
\hline & & & Freq. & 950 & 312 & 38 & & & \\
\hline & & Total & $\%$ & 73.1 & 24.0 & 2.9 & 2.70 & 0.518 & \\
\hline & & Princess & Freq. & 469 & 116 & 15 & & & \\
\hline & & $\begin{array}{l}\text { Nourah bint } \\
\text { Abddulrahman } \\
\text { University }\end{array}$ & $\%$ & 78.2 & 19.3 & 2.5 & 2.76 & 0.484 & \\
\hline & & King Saud & Freq. & 253 & 89 & 8 & 270 & 0506 & \\
\hline & & University & $\%$ & 72.3 & 25.4 & 2.3 & 2.70 & & \\
\hline 12 & $\begin{array}{l}\text { Avording physical } \\
\text { violence with sons. }\end{array}$ & Imam & Freq. & 283 & 55 & 12 & & & 6 \\
\hline & & $\begin{array}{l}\text { Muhammad } \\
\text { ibn Saud } \\
\text { Islamic } \\
\text { University } \\
\end{array}$ & $\%$ & 80.9 & 15.7 & 3.4 & 2.77 & 0.461 & \\
\hline & & Total & Freq. & 1005 & 260 & 35 & 275 & 0.493 & \\
\hline & & Iotal & $\%$ & 77.3 & 20.0 & 2.7 & 2.15 & 0.493 & \\
\hline & & Princess & Freq. & 459 & 130 & 11 & & & \\
\hline & & $\begin{array}{l}\text { Nourah bint } \\
\text { Abddulrahman } \\
\text { University }\end{array}$ & $\%$ & 76.5 & 21.7 & 1.8 & 2.75 & 0.476 & \\
\hline & & King Saud & Freq. & 250 & 93 & 7 & & & \\
\hline & Protecting children & University & $\%$ & 71.4 & 26.6 & 2.0 & 2.69 & 0.503 & \\
\hline 13 & $\begin{array}{l}\text { against the effects } \\
\text { of intellectual }\end{array}$ & Imam & Freq. & 275 & 69 & 6 & & & 8 \\
\hline & deviation's heralds. & $\begin{array}{l}\text { Muhammad } \\
\text { ibn Saud } \\
\text { Islamic } \\
\text { University } \\
\end{array}$ & $\%$ & 78.6 & 19.7 & 1.7 & 2.77 & 0.461 & \\
\hline & & Total & Freq. & 984 & 292 & 24 & 2.74 & 0.480 & \\
\hline & & lotal & $\%$ & 75.7 & 22.5 & 1.8 & 2.14 & 0.480 & \\
\hline & & Princess & Freq. & 453 & 134 & 13 & & & \\
\hline 14 & $\begin{array}{l}\text { Helping children } \\
\text { adapt to social } \\
\text { conditions. }\end{array}$ & $\begin{array}{l}\text { Nourah bint } \\
\text { Abddulrahman } \\
\text { University }\end{array}$ & $\%$ & 75.5 & 22.3 & 2.2 & 2.73 & 0.489 & 10 \\
\hline & & King Saud & Freq. & 250 & 92 & 8 & 2.69 & 0.510 & \\
\hline
\end{tabular}




\begin{tabular}{|c|c|c|c|c|c|c|c|c|c|}
\hline \multirow{7}{*}{ No. } & \multirow{7}{*}{ Iten } & \multirow[b]{3}{*}{ University } & Frequency & \multicolumn{3}{|c|}{ Agreement degree } & \multirow{3}{*}{$\begin{array}{l}\text { Arithmetic } \\
\text { mean }\end{array}$} & \multirow{3}{*}{$\begin{array}{l}\text { Standard } \\
\text { deviation }\end{array}$} & \multirow{7}{*}{ Rank } \\
\hline & & & Percentage & Agree & $\begin{array}{c}\text { Somewhat } \\
\text { agree }\end{array}$ & Disagree & & & \\
\hline & & & $\%$ & 71.4 & 26.3 & 2.3 & & & \\
\hline & & \multirow{2}{*}{$\begin{array}{l}\text { Imam } \\
\text { Muhammad } \\
\text { ibn Saud } \\
\text { Islamic } \\
\text { University } \\
\end{array}$} & Freq. & 272 & 70 & 8 & \multirow[b]{2}{*}{2.75} & \multirow[b]{2}{*}{0.481} & \\
\hline & & & $\%$ & 77.7 & 20.0 & 2.3 & & & \\
\hline & & \multirow{2}{*}{ Total } & Freq. & 975 & 296 & 29 & \multirow{2}{*}{2.73} & \multirow{2}{*}{0.493} & \\
\hline & & & $\%$ & 75.0 & 22.8 & 2.2 & & & \\
\hline \multirow{8}{*}{15} & \multirow{8}{*}{$\begin{array}{l}\text { Upgrading } \\
\text { sincerity in work } \\
\text { among children. }\end{array}$} & \multirow{2}{*}{$\begin{array}{c}\text { Princess } \\
\text { Nourah bint } \\
\text { Abddulrahman } \\
\text { University }\end{array}$} & Freq. & 455 & 136 & 9 & \multirow[b]{2}{*}{2.74} & \multirow[b]{2}{*}{0.470} & \multirow{8}{*}{9} \\
\hline & & & $\%$ & 75.8 & 22.7 & 1.5 & & & \\
\hline & & King Saud & Freq. & 245 & 99 & 6 & 268 & 0.507 & \\
\hline & & University & $\%$ & 70.0 & 28.3 & 1.7 & 2.00 & 0.502 & \\
\hline & & Imam & Freq. & 279 & 66 & 5 & & & \\
\hline & & $\begin{array}{l}\text { ibn Saud } \\
\text { Islamic } \\
\text { University } \\
\end{array}$ & $\%$ & 79.7 & 18.9 & 1.4 & 2.78 & 0.446 & \\
\hline & & \multirow{2}{*}{ Total } & Freq. & 979 & 301 & 20 & \multirow{2}{*}{2.74} & \multirow{2}{*}{0.474} & \\
\hline & & & $\%$ & 75.3 & 23.2 & 1.5 & & & \\
\hline \multirow{8}{*}{16} & \multirow{8}{*}{$\begin{array}{l}\text { Developing social } \\
\text { responsibility } \\
\text { towards the self } \\
\text { and nation among } \\
\text { children. }\end{array}$} & \multirow{2}{*}{$\begin{array}{c}\text { Princess } \\
\text { Nourah bint } \\
\text { Abddulrahman } \\
\text { University }\end{array}$} & Freq. & 451 & 137 & 12 & \multirow[b]{2}{*}{2.73} & \multirow[b]{2}{*}{0.487} & \multirow{8}{*}{11} \\
\hline & & & $\%$ & 75.2 & 22.8 & 2.0 & & & \\
\hline & & King Saud & Freq. & 248 & 94 & 8 & 269 & 0.512 & \\
\hline & & University & $\%$ & 70.9 & 26.9 & 2.3 & 2.09 & 0.512 & \\
\hline & & Imam & Freq. & 275 & 65 & 10 & & & \\
\hline & & $\begin{array}{l}\text { ibn Saud } \\
\text { Islamic } \\
\text { University } \\
\end{array}$ & $\%$ & 78.6 & 18.6 & 2.9 & 2.76 & 0.492 & \\
\hline & & Total & Freq. & 974 & 296 & 30 & 273 & 0405 & \\
\hline & & Toral & $\%$ & 74.9 & 22.8 & 2.3 & 2.15 & 0.493 & \\
\hline & & Princess & Freq. & 455 & 128 & 17 & & & \\
\hline & & $\begin{array}{l}\text { Abddulrahman } \\
\text { University }\end{array}$ & $\%$ & 75.8 & 21.3 & 2.8 & 2.73 & 0.504 & \\
\hline & & King Saud & Freq. & 252 & 91 & 7 & 270 & 0501 & \\
\hline & Developing the & University & $\%$ & 72.0 & 26.0 & 2.0 & 2.10 & 0.501 & \\
\hline 17 & intellectual safety & Imam & Freq. & 277 & 59 & 14 & & & 12 \\
\hline & among children. & $\begin{array}{l}\text { ibn Saud } \\
\text { Islamic } \\
\text { University } \\
\end{array}$ & $\%$ & 79.1 & 16.9 & 4.0 & 2.75 & 0.517 & \\
\hline & & Total & Freq. & 984 & 278 & 38 & 273 & 0.507 & \\
\hline & & Hotal & $\%$ & 75.7 & 21.4 & 2.9 & & 0.001 & \\
\hline & & Genera & mean & & & & 2.75 & & \\
\hline
\end{tabular}

Table 8 illustrates that the agreement of the participants to "the role of woman in implanting moderate Islamic values in children" achieved an arithmetic mean 2.75 out of 3.00 , which was ranked third on the triple scale (from 2.35 out of 3.00 ), indicating its achievement. Accordingly, there is consistency of their agreement, reaching an arithmetic mean 2.70 to 2.86. They agreed on seventeen roles, the most significant of which are $(7,5,3,10$, and 4$)$, as follows:
1. Item (7) "educating children about the rules and morals of Islam" was ranked first, with an arithmetic mean 2.86 out of 4. The female students of Imam Muhammad ibn Saud Islamic University were ranked the highest with an arithmetic mean 2.89 out of 4; followed by those of Princess Nourah bint Abddulrahman University, with an arithmetic mean 2.87 out of 4; and those of King Saud University, with an arithmetic mean 2.83 out of 4 . 
2. Item (5) "motivating children to adhere to social morals" was ranked second, with an arithmetic mean 2.84 out of 4 . The female students of Imam Muhammad ibn Saud Islamic University were ranked the highest with an arithmetic mean 2.86 out of 4 ; followed by those of Princess Nourah bint Abddulrahman University, with an arithmetic mean 2.85 out of 4; and those of King Saud University, with an arithmetic mean 2.82 out of 4 .

3. Item (3) "illustrating the prohibition of self-aggression and avoiding injustice" was ranked third, with an arithmetic mean 2.80 out of 4 . The female students of Imam Muhammad ibn Saud Islamic University were ranked the highest with an arithmetic mean 2.82 out of 4; followed by those of Princess Nourah bint Abddulrahman University, with an arithmetic mean 2.81 out of 4; and those of King Saud University, with an arithmetic mean 2.79 out of 4 .

4. Item (10) "children avoid the manifestation of behavioral deviation" was ranked fourth, with an arithmetic mean 2.78 out of 4 . The female students of Imam Muhammad ibn Saud Islamic University were ranked the highest with an arithmetic mean 2.80 out of 4; followed by those of Princess Nourah bint
Abddulrahman University, with an arithmetic mean 2.78 out of 4; and those of King Saud University, with an arithmetic mean 2.75 out of 4 .

5. Item (4) "linking children to good friends" was ranked fifth, with an arithmetic mean 2.77 out of 4 . The female students of Imam Muhammad ibn Saud Islamic University were ranked the highest with an arithmetic mean 2.81 out of 4 ; followed by those of Princess Nourah bint Abddulrahman University, with an arithmetic mean 2.78 out of 4; and those of King Saud University, with an arithmetic mean 2.73 out of 4 .

Results indicate that the most significant role of woman in implanting moderate Islamic values in children is to educate children about the rules and ethics of Islam, because the mother is keen on bringing up children in a moderate Islamic way and educates them the Islamic rules and ethics.

These results agree with Elqadeb (2010) focusing on moderation values of Islam; Elshahrany (2009) reporting that education on moderation is an important aspect that should be stressed to achieve intellectual safety; and Elashqer (2010) addressing the role of teachers and principals in consolidating moderation of the students to protect them from intellectual extremism.

Table 9. Responses of the participants to the role of woman in reinforcing, respecting, and improving the self and maintaining cultural distinctiveness

\begin{tabular}{|c|c|c|c|c|c|c|c|c|c|}
\hline \multirow{2}{*}{ No. } & \multirow{2}{*}{\multicolumn{2}{|c|}{ Item }} & Frequency & \multicolumn{3}{|c|}{ Agreement degree } & \multirow{2}{*}{$\begin{array}{l}\text { Arithmetic } \\
\text { mean }\end{array}$} & \multirow{2}{*}{$\begin{array}{l}\text { Standard } \\
\text { deviation }\end{array}$} & \multirow{2}{*}{ Rank } \\
\hline & & & Percentage & Yes & Somewhat & No & & & \\
\hline \multirow{8}{*}{1} & \multirow{8}{*}{$\begin{array}{l}\text { Helping children pride } \\
\text { themselves on boasting the } \\
\text { culture and civilization of } \\
\text { community }\end{array}$} & \multirow{2}{*}{$\begin{array}{l}\text { Princess Nourah bint } \\
\text { Abddulrahman } \\
\text { University } \\
\end{array}$} & Freq. & 378 & 212 & 10 & \multirow{2}{*}{2.61} & \multirow{2}{*}{0.521} & \multirow{8}{*}{5} \\
\hline & & & $\%$ & 63.0 & 35.3 & 1.7 & & & \\
\hline & & \multirow{2}{*}{ King Saud University } & Freq. & 198 & 145 & 7 & \multirow{2}{*}{2.55} & \multirow{2}{*}{0.537} & \\
\hline & & & $\%$ & 56.6 & 41.4 & 2.0 & & & \\
\hline & & \multirow{2}{*}{$\begin{array}{c}\text { Imam Muhammad ibn } \\
\text { Saud Islamic } \\
\text { University } \\
\end{array}$} & Freq. & 237 & 107 & 6 & \multirow{2}{*}{2.66} & \multirow{2}{*}{0.509} & \\
\hline & & & $\%$ & 67.7 & 30.6 & 1.7 & & & \\
\hline & & \multirow{2}{*}{ Total } & Freq. & 813 & 464 & 23 & \multirow{2}{*}{2.61} & \multirow{2}{*}{0.523} & \\
\hline & & & $\%$ & 62.5 & 35.7 & 1.8 & & & \\
\hline \multirow{8}{*}{2} & \multirow{8}{*}{$\begin{array}{l}\text { Discussing children on } \\
\text { issues of interest }\end{array}$} & \multirow{2}{*}{$\begin{array}{l}\text { Princess Nourah bint } \\
\text { Abddulrahman } \\
\text { University } \\
\end{array}$} & Freq. & 329 & 251 & 20 & \multirow{2}{*}{2.51} & \multirow{2}{*}{0.563} & \multirow{8}{*}{13} \\
\hline & & & $\%$ & 54.8 & 41.8 & 3.3 & & & \\
\hline & & \multirow{2}{*}{ King Saud University } & Freq. & 174 & 163 & 13 & \multirow{2}{*}{2.46} & \multirow{2}{*}{0.569} & \\
\hline & & & $\%$ & 49.7 & 46.6 & 3.7 & & & \\
\hline & & \multirow{2}{*}{$\begin{array}{c}\text { Imam Muhammad ibn } \\
\text { Saud Islamic } \\
\text { University } \\
\end{array}$} & Freq. & 210 & 128 & 12 & & & \\
\hline & & & $\%$ & 60.0 & 36.6 & 3.4 & 2.57 & 0.561 & \\
\hline & & & Freq. & 713 & 542 & 45 & & & \\
\hline & & Total & $\%$ & 54.8 & 41.7 & 3.5 & 2.51 & 0.565 & \\
\hline & & Princess Nourah bint & Freq. & 372 & 201 & 27 & & & \\
\hline & & $\begin{array}{c}\text { Abddulrahman } \\
\text { University }\end{array}$ & $\%$ & 62.0 & 33.5 & 4.5 & 2.58 & 0.579 & \\
\hline & Educating children about & King Saud University & Freq. & 192 & 144 & 14 & 251 & 0.575 & \\
\hline 3 & strengths and consolidating & King Saud University & $\%$ & 54.9 & 41.1 & 4.0 & 2.51 & 0.575 & 8 \\
\hline & them & Imam Muhammad ibn & Freq. & 232 & 100 & 18 & & & \\
\hline & & $\begin{array}{c}\text { Saud Islamic } \\
\text { University }\end{array}$ & $\%$ & 66.3 & 28.6 & 5.1 & 2.61 & 0.584 & \\
\hline & & Total & Freq. & 796 & 445 & 59 & 2.57 & 0.580 & \\
\hline
\end{tabular}




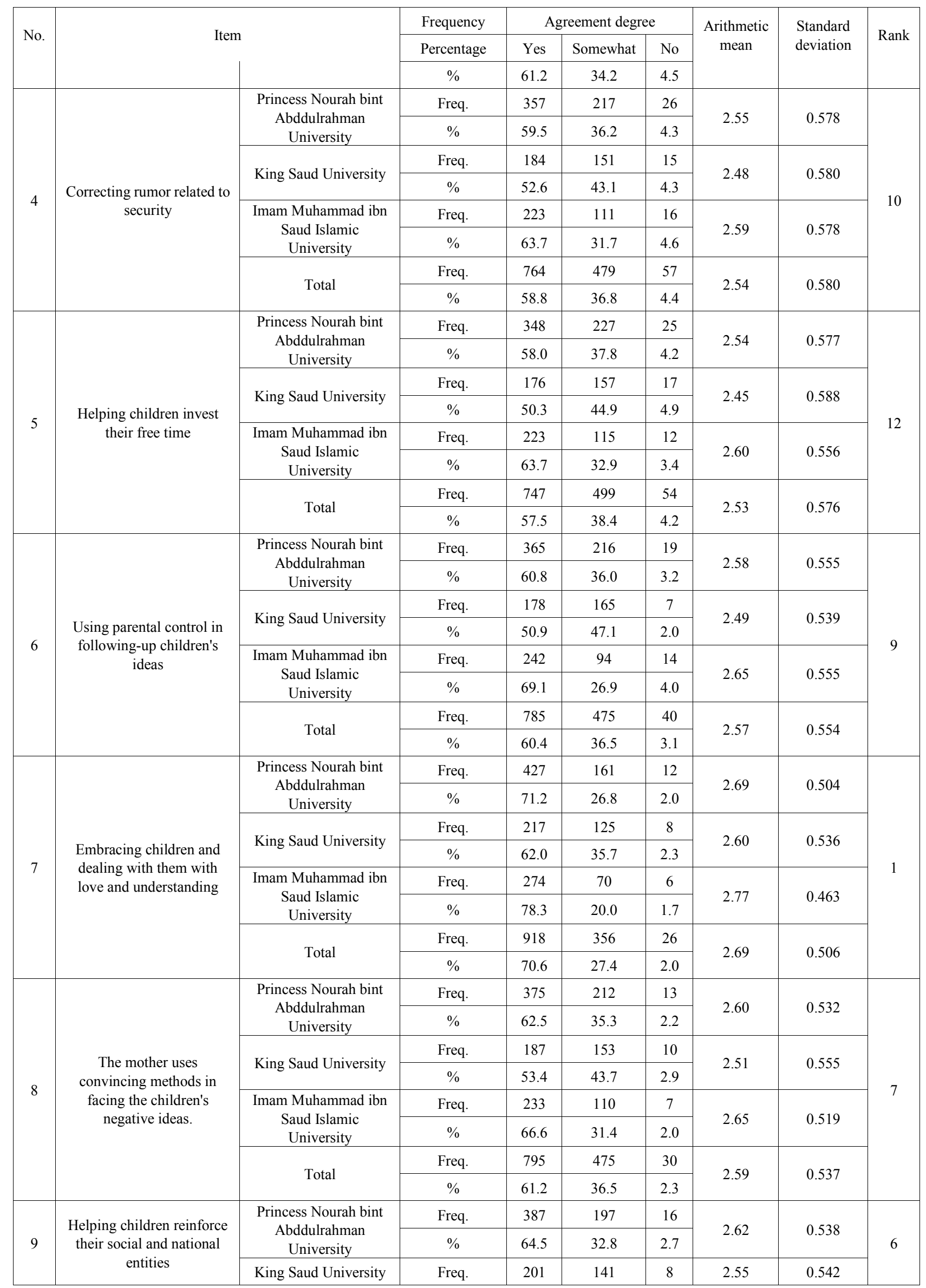




\begin{tabular}{|c|c|c|c|c|c|c|c|c|c|}
\hline \multirow{7}{*}{ No. } & \multirow{3}{*}{\multicolumn{2}{|c|}{ Item }} & Frequency & \multicolumn{3}{|c|}{ Agreement degree } & \multirow{3}{*}{$\begin{array}{l}\text { Arithmetic } \\
\text { mean }\end{array}$} & \multirow{3}{*}{$\begin{array}{l}\text { Standard } \\
\text { deviation }\end{array}$} & \multirow{7}{*}{ Rank } \\
\hline & & & Percentage & Yes & Somewhat & No & & & \\
\hline & & & $\%$ & 57.4 & 40.3 & 2.3 & & & \\
\hline & & \multirow{2}{*}{$\begin{array}{c}\text { Imam Muhammad ibn } \\
\text { Saud Islamic } \\
\text { University }\end{array}$} & Freq. & 239 & 100 & 11 & \multirow{2}{*}{2.65} & \multirow{2}{*}{0.539} & \\
\hline & & & $\%$ & 68.3 & 28.6 & 3.1 & & & \\
\hline & & \multirow{2}{*}{ Total } & Freq. & 827 & 438 & 35 & \multirow{2}{*}{2.61} & \multirow{2}{*}{0.540} & \\
\hline & & & $\%$ & 63.6 & 33.7 & 2.7 & & & \\
\hline \multirow{8}{*}{10} & \multirow{8}{*}{$\begin{array}{l}\text { Guiding children to the } \\
\text { effective use of technology }\end{array}$} & \multirow{2}{*}{$\begin{array}{l}\text { Princess Nourah bint } \\
\text { Abddulrahman } \\
\text { University }\end{array}$} & Freq. & 356 & 213 & 31 & \multirow{2}{*}{2.54} & \multirow{2}{*}{0.593} & \multirow{8}{*}{11} \\
\hline & & & $\%$ & 59.3 & 35.5 & 5.2 & & & \\
\hline & & \multirow{2}{*}{ King Saud University } & Freq. & 180 & 157 & 13 & \multirow{2}{*}{2.48} & \multirow{2}{*}{0.570} & \\
\hline & & & $\%$ & 51.4 & 44.9 & 3.7 & & & \\
\hline & & \multirow{2}{*}{$\begin{array}{c}\text { Imam Muhammad ibn } \\
\text { Saud Islamic } \\
\text { University }\end{array}$} & Freq. & 231 & 95 & 24 & \multirow{2}{*}{2.72} & \multirow{2}{*}{0.509} & \\
\hline & & & $\%$ & 66.0 & 27.1 & 6.9 & & & \\
\hline & & $T$ & Freq. & 767 & 465 & 68 & $2=0$ & 0505 & \\
\hline & & 10tal & $\%$ & 59.0 & 35.8 & 5.2 & 2.04 & 0.070 & \\
\hline & & Princess Nourah bint & Freq. & 418 & 162 & 20 & 266 & $0=20$ & \\
\hline & & University & $\%$ & 69.7 & 27.0 & 3.3 & 2.00 & 0.039 & \\
\hline & & $Y$ inc Soud Uningit & Freq. & 215 & 120 & 15 & 257 & 0576 & \\
\hline 11 & Helping and encouraging & King saud University & $\%$ & 61.4 & 34.3 & 4.3 & 2.37 & $0.5 / 6$ & $A$ \\
\hline 11 & to serve community & Imam Muhammad ibn & Freq. & 262 & 78 & 10 & 72 & 0500 & 4 \\
\hline & & University & $\%$ & 74.9 & 22.3 & 2.9 & 2.12 & 0.509 & \\
\hline & & 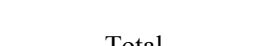 & Freq. & 895 & 360 & 45 & $265+2+3$ & 0544 & \\
\hline & & 10tal & $\%$ & 68.8 & 27.7 & 3.5 & 2.05 & 0.544 & \\
\hline & & Princess Nourah bint & Freq. & 434 & 147 & 19 & 60 & 0506 & \\
\hline & & $\begin{array}{l}\text { Abaduiranman } \\
\text { University }\end{array}$ & $\%$ & 72.3 & 24.5 & 3.2 & 2.09 & 0.520 & \\
\hline & & $Y$ in $\mathrm{C}$ - d Uni & Freq. & 227 & 111 & 12 & 261 & 0554 & \\
\hline 12 & Helping children take good & Ning saud Unversily & $\%$ & 64.9 & 31.7 & 3.4 & 2.01 & 0.534 & 0 \\
\hline 12 & models & Imam Muhammad ibn & Freq. & 269 & 70 & 11 & 77 & $0-07+$ & 2 \\
\hline & & $\begin{array}{l}\text { Saud Islamic } \\
\text { University }\end{array}$ & $\%$ & 76.9 & 20.0 & 3.1 & 2.14 & 0.501 & \\
\hline & & $T$ & Freq. & 930 & 328 & 42 & 260 & 0520 & \\
\hline & & Iotal & $\%$ & 71.5 & 25.2 & 3.2 & 2.68 & 0.530 & \\
\hline & & Princess Nourah bint & Freq. & 425 & 160 & 15 & 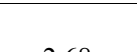 & $0-17$ & \\
\hline & & $\begin{array}{c}\text { Abddulrahman } \\
\text { University }\end{array}$ & $\%$ & 70.8 & 26.7 & 2.5 & 2.68 & 0.517 & \\
\hline & & 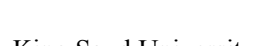 & Freq. & 218 & 122 & 10 & 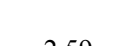 & $0=57$ & \\
\hline 12 & Providing a family & King Saud Unıversity & $\%$ & 62.3 & 34.9 & 2.9 & 2.59 & 0.547 & 2 \\
\hline 13 & $\begin{array}{l}\text { environment characterized } \\
\text { by strong communication }\end{array}$ & Imam Muhammad ibn & Freq. & 273 & 70 & 7 & 76 & 0477 & 3 \\
\hline & & $\begin{array}{l}\text { Saud Islamic } \\
\text { University }\end{array}$ & $\%$ & 78.0 & 20.0 & 2.0 & 2.76 & 0.472 & \\
\hline & & $T=1$ & Freq. & 916 & 352 & 32 & $\log ^{-1}$ & $0-017$ & \\
\hline & & lotal & $\%$ & 70.5 & 27.1 & 2.5 & 2.68 & 0.517 & \\
\hline & & General mean & & & & & 2.60 & 0.39 & \\
\hline
\end{tabular}

Table 9 illustrates that the agreement of the participants to "the role of woman in reinforcing, respecting, and improving the self and maintaining cultural distinctiveness" achieved an arithmetic mean 2.60 out of 3.00 , which was ranked third on the triple scale (from 2.35 out of 3.00), indicating its achievement.
Accordingly, there is consistency of their agreement, reaching an arithmetic mean 2.51 to 2.69 . They agreed on thirteen roles, the most significant of which are $(7,12,13,11$, and 1) in a descending order, as follows:

1. Item (7) "embracing children and dealing with them with love and understanding" was ranked first, with an 
arithmetic mean 2.69 out of 4 . The female students of Imam Muhammad ibn Saud Islamic University were ranked the highest with an arithmetic mean 2.77 out of 4; followed by those of Princess Nourah bint Abddulrahman University, with an arithmetic mean 2.69 out of 4; and those of King Saud University, with an arithmetic mean 2.60 out of 4 .

2. Item (12) "helping children take good models" was ranked second, with an arithmetic mean 2.68 out of 4 . The female students of Imam Muhammad ibn Saud Islamic University were ranked the highest with an arithmetic mean 2.74 out of 4; followed by those of Princess Nourah bint Abddulrahman University, with an arithmetic mean 2.69 out of 4; and those of King Saud University, with an arithmetic mean 2.61 out of 4 .

3. Item (13) "providing a family environment characterized by strong communication" was ranked third, with arithmetic mean 2.68 out of 4 . The female students of Imam Muhammad ibn Saud Islamic University were ranked the highest with an arithmetic mean 2.76 out of 4 ; followed by those of Princess Nourah bint Abddulrahman University, with an arithmetic mean 2.68 out of 4; and those of King Saud University, with an arithmetic mean 2.59 out of 4 .

4. Item (11) "helping and encouraging children to achieve success to serve community" was ranked fourth, with an arithmetic mean 2.65 out of 4 . The female students of Imam Muhammad ibn Saud Islamic University were ranked the highest with an arithmetic mean 2.72 out of 4; followed by those of Princess Nourah bint Abddulrahman University, with an arithmetic mean 2.66 out of 4; and those of King Saud University, with an arithmetic mean 2.57 out of 4 .

5. Item (1) "Helping children pride themselves on boasting the culture and civilization of community" was ranked fifth, with an arithmetic mean 2.61 out of 4 . The female students of Imam Muhammad ibn Saud Islamic University were ranked the highest with an arithmetic mean 2.66 out of 4; followed by those of Princess Nourah bint Abddulrahman University, with an arithmetic mean 2.61 out of 4; and those of King Saud University, with an arithmetic mean 2.55 out of 4 .

Results indicate that the most significant role of woman in reinforcing, respecting, and improving the self and maintaining cultural distinctiveness is embracing children and dealing with them with love and understanding because the mother always seeks to provide a sound family environment to children.

These results are consistent with Elshahrany (2009) indicating that intellectual safety is closely related to the social, cultural, intellectual, economic, religious, and psychological aspects of life. They are also consistent with Elhawaish (2011), reporting that intellectual protection, security awareness, and protecting the original components of culture have a close relation to the various aspects of life, e.g. protecting them from the traps of intellectual deviation.

Table 10. Responses of the participants to the items of "the role of woman in bringing up children on respecting freedoms" domain in a descending order according to agreement

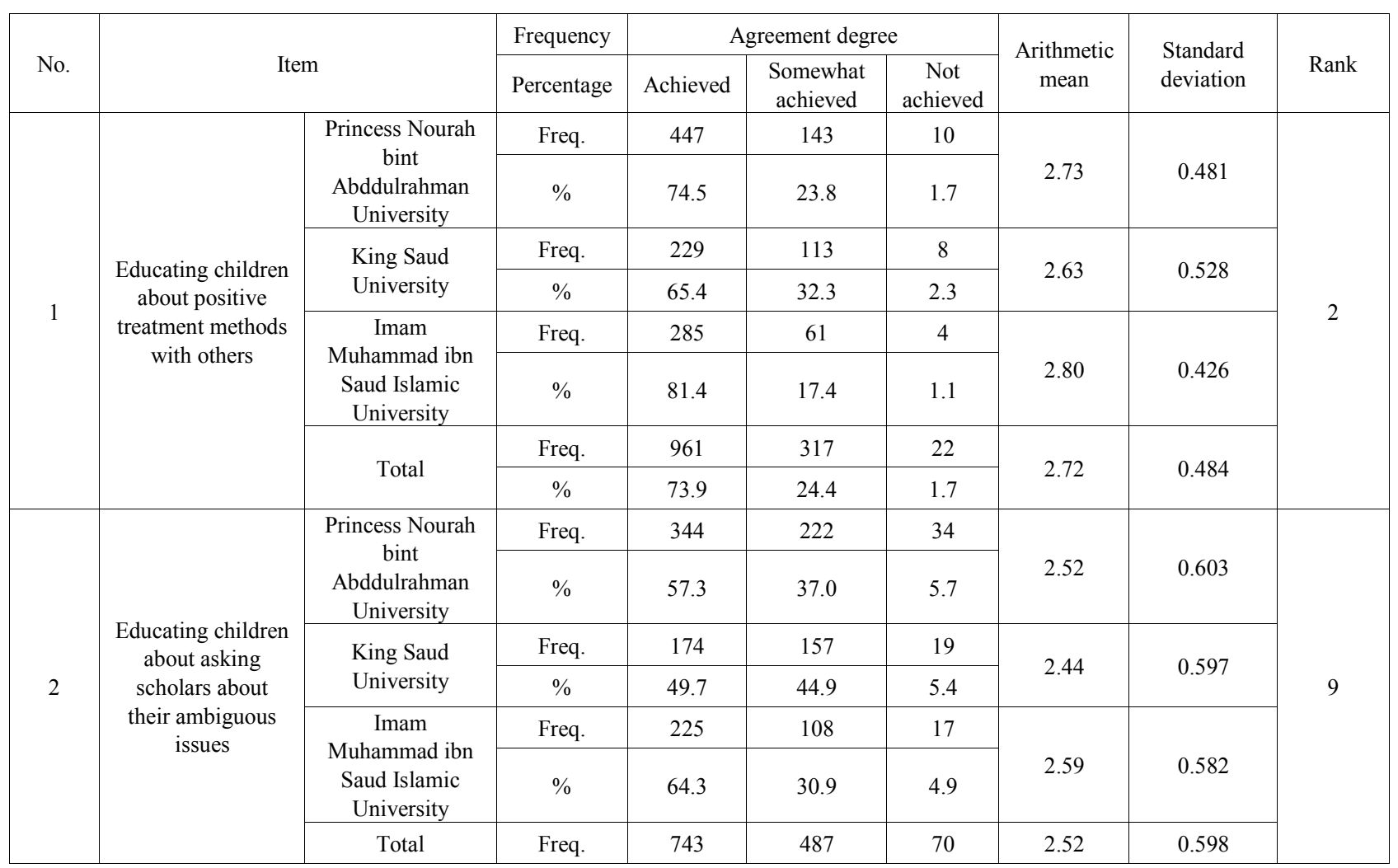




\begin{tabular}{|c|c|c|c|c|c|c|c|c|c|}
\hline \multirow{3}{*}{ No. } & \multirow{3}{*}{\multicolumn{2}{|c|}{ Item }} & Frequency & \multicolumn{3}{|c|}{ Agreement degree } & \multirow{3}{*}{$\begin{array}{l}\text { Arithmetic } \\
\text { mean }\end{array}$} & \multirow{3}{*}{$\begin{array}{l}\text { Standard } \\
\text { deviation }\end{array}$} & \multirow{3}{*}{ Rank } \\
\hline & & & Percentage & Achieved & $\begin{array}{l}\text { Somewhat } \\
\text { achieved }\end{array}$ & $\begin{array}{c}\text { Not } \\
\text { achieved }\end{array}$ & & & \\
\hline & & & $\%$ & 57.2 & 37.5 & 5.4 & & & \\
\hline \multirow{8}{*}{3} & \multirow{8}{*}{$\begin{array}{l}\text { Developing the } \\
\text { culture of } \\
\text { respecting others' } \\
\text { opinions }\end{array}$} & \multirow{2}{*}{$\begin{array}{c}\text { Princess Nourah } \\
\text { bint } \\
\text { Abddulrahman } \\
\text { University } \\
\end{array}$} & Freq. & 389 & 189 & 22 & \multirow[b]{2}{*}{2.61} & \multirow[b]{2}{*}{0.558} & \multirow{8}{*}{7} \\
\hline & & & $\%$ & 64.8 & 31.5 & 3.7 & & & \\
\hline & & King Saud & Freq. & 194 & 141 & 15 & & & \\
\hline & & University & $\%$ & 55.4 & 40.3 & 4.3 & 2.51 & 0.500 & \\
\hline & & Imam & Freq. & 253 & 88 & 9 & & & \\
\hline & & $\begin{array}{c}\text { Saud Islamic } \\
\text { University }\end{array}$ & $\%$ & 72.3 & 25.1 & 2.6 & 2.70 & 0.513 & \\
\hline & & \multirow{2}{*}{ Total } & Freq. & 836 & 418 & 46 & \multirow{2}{*}{2.61} & \multirow{2}{*}{0.556} & \\
\hline & & & $\%$ & 64.3 & 32.2 & 3.5 & & & \\
\hline \multirow{8}{*}{4} & \multirow{8}{*}{$\begin{array}{l}\text { Illustrating that } \\
\text { Allah prohibited } \\
\text { killing the soul } \\
\text { except by legal } \\
\text { right }\end{array}$} & \multirow{2}{*}{$\begin{array}{c}\text { Princess Nourah } \\
\text { bint } \\
\text { Abddulrahman } \\
\text { University } \\
\end{array}$} & Freq. & 466 & 119 & 15 & \multirow[b]{2}{*}{2.75} & \multirow[b]{2}{*}{0.487} & \multirow{8}{*}{1} \\
\hline & & & $\%$ & 77.7 & 19.8 & 2.5 & & & \\
\hline & & King Saud & Freq. & 241 & 99 & 10 & 266 & 0531 & \\
\hline & & University & $\%$ & 68.9 & 28.3 & 2.9 & 2.00 & . & \\
\hline & & Imam & Freq. & 287 & 57 & 6 & & & \\
\hline & & $\begin{array}{c}\text { Saud Islamic } \\
\text { University }\end{array}$ & $\%$ & 82.0 & 16.3 & 1.7 & 2.80 & 0.439 & \\
\hline & & \multirow{2}{*}{ Total } & Freq. & 994 & 275 & 31 & \multirow{2}{*}{2.74} & \multirow{2}{*}{0.490} & \\
\hline & & & $\%$ & 76.5 & 21.2 & 2.4 & & & \\
\hline \multirow{8}{*}{5} & & Princess Nourah & Freq. & 452 & 135 & 13 & & & \\
\hline & & $\begin{array}{c}\text { Abddulrahman } \\
\text { University }\end{array}$ & $\%$ & 75.3 & 22.5 & 2.2 & 2.73 & 0.490 & \\
\hline & & King Saud & Freq. & 237 & 102 & 11 & 265 & 0544 & \\
\hline & Guiding children & University & $\%$ & 67.7 & 29.1 & 3.1 & 2.05 & 0.041 & 2 \\
\hline & humiliate others & Imam & Freq. & 280 & 65 & 5 & & & 3 \\
\hline & & $\begin{array}{c}\text { Saud Islamic } \\
\text { University }\end{array}$ & $\%$ & 80.0 & 18.6 & 1.4 & 2.79 & 0.444 & \\
\hline & & Total & Freq. & 969 & 302 & 29 & 277 & 0405 & \\
\hline & & Total & $\%$ & 74.5 & 23.2 & 2.2 & 2.12 & 0.495 & \\
\hline & & Princess Nourah & Freq. & 411 & 171 & 18 & & & \\
\hline & & $\begin{array}{c}\text { Abddulrahman } \\
\text { University }\end{array}$ & $\%$ & 68.5 & 28.5 & 3.0 & 2.66 & 0.535 & \\
\hline & & King Saud & Freq. & 207 & 131 & 12 & 56 & 0560 & \\
\hline 6 & Helping children & University & $\%$ & 59.1 & 37.4 & 3.4 & 2.00 & 0.502 & \\
\hline 0 & $\begin{array}{l}\text { failure } \\
\text { face }\end{array}$ & Imam & Freq. & 263 & 80 & 7 & & & 5 \\
\hline & & $\begin{array}{c}\text { Saud Islamic } \\
\text { University }\end{array}$ & $\%$ & 75.1 & 22.9 & 2.0 & 2.73 & 0.487 & \\
\hline & & Total & Freq. & 881 & 382 & 37 & 265 & 0534 & \\
\hline & & IOtal & $\%$ & 67.8 & 29.4 & 2.8 & 2.05 & 0.534 & \\
\hline & & Princess Nourah & Freq. & 381 & 200 & 19 & & & \\
\hline 7 & $\begin{array}{l}\text { Guiding children } \\
\text { to respect the other }\end{array}$ & $\begin{array}{c}\text { Abddulrahman } \\
\text { University }\end{array}$ & $\%$ & 63.5 & 33.3 & 3.2 & 2.60 & 0.551 & 8 \\
\hline & cultures & King Saud & Freq. & 194 & 145 & 11 & 252 & 0560 & \\
\hline & & University & $\%$ & 55.4 & 41.4 & 3.1 & 2.02 & 0.500 & \\
\hline
\end{tabular}




\begin{tabular}{|c|c|c|c|c|c|c|c|c|c|}
\hline \multirow{6}{*}{ No. } & \multirow{2}{*}{\multicolumn{2}{|c|}{ Item }} & Frequency & \multicolumn{3}{|c|}{ Agreement degree } & \multirow{2}{*}{$\begin{array}{l}\text { Arithmetic } \\
\text { mean }\end{array}$} & \multirow{2}{*}{$\begin{array}{l}\text { Standard } \\
\text { deviation }\end{array}$} & \multirow{6}{*}{ Rank } \\
\hline & & & Percentage & Achieved & $\begin{array}{c}\text { Somewhat } \\
\text { achieved }\end{array}$ & $\begin{array}{c}\text { Not } \\
\text { achieved }\end{array}$ & & & \\
\hline & & \multirow{2}{*}{$\begin{array}{c}\text { Imam } \\
\text { Muhammad ibn } \\
\text { Saud Islamic } \\
\text { University }\end{array}$} & Freq. & 244 & 96 & 10 & \multirow[b]{2}{*}{2.67} & \multirow[b]{2}{*}{0.529} & \\
\hline & & & $\%$ & 69.7 & 27.4 & 2.9 & & & \\
\hline & & \multirow{2}{*}{ Total } & Freq. & 819 & 441 & 40 & \multirow{2}{*}{2.60} & \multirow{2}{*}{0.549} & \\
\hline & & & $\%$ & 63.0 & 33.9 & 3.1 & & & \\
\hline \multirow{8}{*}{8} & \multirow{8}{*}{$\begin{array}{l}\text { Guiding children } \\
\text { to benefit from } \\
\text { positive opinions }\end{array}$} & \multirow{2}{*}{$\begin{array}{c}\text { Princess Nourah } \\
\text { bint } \\
\text { Abddulrahman } \\
\text { University } \\
\end{array}$} & Freq. & 433 & 148 & 19 & \multirow[b]{2}{*}{2.69} & \multirow[b]{2}{*}{0.527} & \multirow{8}{*}{4} \\
\hline & & & $\%$ & 72.2 & 24.7 & 3.2 & & & \\
\hline & & King Saud & Freq. & 217 & 119 & 14 & 58 & 0570 & \\
\hline & & University & $\%$ & 62.0 & 34.0 & 4.0 & 2.50 & 0.570 & \\
\hline & & Imam & Freq. & 276 & 66 & 8 & & & \\
\hline & & $\begin{array}{c}\text { Saud Islamic } \\
\text { University }\end{array}$ & $\%$ & 78.9 & 18.9 & 2.3 & 2.77 & 0.475 & \\
\hline & & \multirow{2}{*}{ Total } & Freq. & 926 & 333 & 41 & \multirow{2}{*}{2.68} & \multirow{2}{*}{0.530} & \\
\hline & & & $\%$ & 71.2 & 25.6 & 3.2 & & & \\
\hline \multirow{8}{*}{9} & \multirow{8}{*}{$\begin{array}{c}\text { Helping children } \\
\text { adopt purposeful } \\
\text { ideas to serve } \\
\text { community }\end{array}$} & \multirow{2}{*}{$\begin{array}{c}\text { Princess Nourah } \\
\text { bint } \\
\text { Abddulrahman } \\
\text { University } \\
\end{array}$} & Freq. & 403 & 173 & 24 & \multirow[b]{2}{*}{2.63} & \multirow[b]{2}{*}{0.560} & \multirow{8}{*}{6} \\
\hline & & & $\%$ & 67.2 & 28.8 & 4.0 & & & \\
\hline & & \multirow{2}{*}{$\begin{array}{l}\text { King Saud } \\
\text { University }\end{array}$} & Freq. & 212 & 121 & 17 & \multirow{2}{*}{2.56} & \multirow{2}{*}{0.587} & \\
\hline & & & $\%$ & 60.6 & 34.6 & 4.9 & & & \\
\hline & & \multirow{2}{*}{$\begin{array}{c}\text { Imam } \\
\text { Muhammad ibn } \\
\text { Saud Islamic } \\
\text { University }\end{array}$} & Freq. & 249 & 92 & 9 & \multirow[b]{2}{*}{2.69} & \multirow[b]{2}{*}{0.517} & \\
\hline & & & $\%$ & 71.1 & 26.3 & 2.6 & & & \\
\hline & & Toto & Freq. & 864 & 386 & 50 & 263 & 0550 & \\
\hline & & Iotal & $\%$ & 66.5 & 29.7 & 3.8 & 2.03 & 0.538 & \\
\hline & & Gen & 1 mean & & & & 2.65 & & \\
\hline
\end{tabular}

Table 10 illustrates that the agreement of the participants to " the role of woman in bringing up children on respecting the freedom of others" achieved an arithmetic mean 2.65 out of 3.00, which was ranked third on the triple scale (from 2.35 to 3.00 ), indicating its achievement.

Accordingly, there is consistency of their agreement, reaching an arithmetic mean from 2.52 to 2.74 . They agreed on nine roles, the most significant of which were $(4,1,5,8$, and 6) in a descending order, as follows:

1. Item (4) "illustrating that Allah prohibited killing the soul except by legal right" was ranked first, with an arithmetic mean 2.74 out of 4 . The female students of Imam Muhammad ibn Saud Islamic University were ranked the highest with an arithmetic mean 2.80 out of 4 ; followed by those of Princess Nourah bint Abddulrahman University, with an arithmetic mean 2.75 out of 4; and those of King Saud University, with an arithmetic mean 2.66 out of 4 .

2. Item (1) "educating children on positive treatment methods with others" was ranked second, with an arithmetic mean 2.72 out of 4 . The female students of Imam Muhammad ibn Saud Islamic University were ranked the highest with an arithmetic mean 2.80 out of 4; followed by those of Princess Nourah bint Abddulrahman University, with an arithmetic mean 2.73 out of 4; and those of King Saud University, with an arithmetic mean 2.63 out of 4 .

3. Item (5) "guiding children not to mock and humiliate others" was ranked third, with an arithmetic mean 2.72 out of 4. The female students of Imam Muhammad ibn Saud Islamic University were ranked the highest with an arithmetic mean 2.79 out of 4 ; followed by those of Princess Nourah bint Abddulrahman University, with an arithmetic mean 2.73 out of 4; and those of King Saud University, with an arithmetic mean 2.65 out of 4 . 4. Item (8) "guiding children to benefit from positive opinions" was ranked fourth, with an arithmetic mean 2.68 out of 4 . The female students of Imam Muhammad ibn Saud Islamic University were ranked the highest with an arithmetic mean 2.77 out of 4 ; followed by those of Princess Nourah bint Abddulrahman University, with an arithmetic mean 2.69 out of 4; and those of King Saud University, with an arithmetic mean 2.58 out of 4 . 
5. Item (6) "helping children face situations of failure" was ranked fifth, with an arithmetic mean 2.65 out of 4 . The female students of Imam Muhammad ibn Saud Islamic University were ranked the highest with an arithmetic mean 2.73 out of 4 ; followed by those of Princess Nourah bint Abddulrahman University, with an arithmetic mean 2.66 out of 4; and those of King Saud University, with an arithmetic mean 2.56 out of 4 .

Results indicate that the most significant roles of woman in bringing up children on respecting the freedom of others are "illustrating that Allah prohibited killing the soul except by legal right" because the mother often seeks to her children being affected with wrong ideas that disseminated lately.

This result is consistent with Elhaider (2002) illustrating that social institutions have a consolidated role in facing the violations of intellectual safety; Ameen (2012) reporting the role of woman in education, implanting positive ideas, and reinforcing nationalism of children and youth to counter passivism and terrorism; and Alotaibi (2007) addressing the role of family, especially the mother, in taking a good moral that aims to implant ethical morals, educate children to purposeful dialogue, respecting opinions and freedoms, and disseminating family dialogue to illustrate that Allah prohibited killing the soul except by legal right

They also assure the role of mosque in religious enlightenment that corresponds to the variable life conditions and current actions according to a moderate methodology that focuses on the principles and applications of moderation.

They also address the role of mass media in transferring social heritage, purifying it from misleading ideas and bad habits, assuring freedom respect and not to infringe others' freedom.

\section{Results}

I. Results related to the first question regarding the role of woman in bringing up children according to the culture and mechanism of family dialogue, in a descending order as follows:

1. "The mother educates children", with an arithmetic mean 2.64 out of 4, where the female students of Imam Muhammad ibn Saud Islamic University were ranked the highest in agreement with an arithmetic mean 2.67 out of 4; followed by those of Princess Nourah bint Abddulrahman University, with an arithmetic mean 2.65 out of 4; and those of King Saud University, with an arithmetic mean 2.57 out of 4 .

2. "The mother uses dialogue to implement morals and ethics, with an arithmetic mean 2.64 out of 4 , where the female students of Imam Muhammad ibn Saud Islamic University were ranked the highest in agreement with an arithmetic mean 2.72 out of 4 ; followed by those of Princess Nourah bint Abddulrahman University, with an arithmetic mean 2.65 out of 4 ; and those of King
Saud University, with an arithmetic mean 2.53 out of 4 .

$3 . \quad$ "The mother corrects wrong concepts among children", with an arithmetic mean 2.63 out of 4 , where the female students of Imam Muhammad ibn Saud Islamic University were ranked the highest in agreement with an arithmetic mean 2.71 out of 4 ; followed by those of Princess Nourah bint Abddulrahman University, with an arithmetic mean 2.64 out of 4; and those of King Saud University, with an arithmetic mean 2.53 out of 4 .

4. "The mother is interested in solving problems and disagreements among children", with an arithmetic mean 2.58 out of 4 , where the female students of Imam Muhammad ibn Saud Islamic University were ranked the highest in agreement with an arithmetic mean 2.67 out of 4; followed by those of Princess Nourah bint Abddulrahman University, with an arithmetic mean 2.60 out of 4; and those of King Saud University, with an arithmetic mean 2.47 out of 4 .

5. "Introducing the legal rules regulating the relationship between the ruler and the ruled", with an arithmetic mean 2.57 out of 4 , where the female students of Imam Muhammad ibn Saud Islamic University were ranked the highest in agreement with an arithmetic mean 2.61 out of 4; followed by those of Princess Nourah bint Abddulrahman University, with an arithmetic mean 2.58 out of 4, and those of King Saud University, with an arithmetic mean 2.52 out of 4 .

The most significant roles of woman in bringing up children according to the culture and mechanism of family dialogue are that she "educates children". This is interpreted that she is keen on bringing them up in a way they adopt with community by acquiring the permanent community culture.

\section{Results related to the second question regarding the role of woman in implanting moderate Islamic values in children in a descending order, as follows:}

The participants agreed on" the role of woman in implanting moderate Islamic values in children", with an arithmetic mean of (2.75 out of 3.00).

The participants agreed on seventeen roles of the woman in implanting moderate Islamic values in children", as follows:

1. "Educating children about the rules and morals of Islam", with an arithmetic mean 2.86 out of 4 , where the female students of Imam Muhammad ibn Saud Islamic University were ranked the highest in agreement with an arithmetic mean 2.89 out of 4 ; followed by those of Princess Nourah bint Abddulrahman University, with an arithmetic mean 2.87 out of 4 , and those of King Saud University, with an arithmetic mean 2.83 out of 4 .

2. "Motivating children to adhere to social morals", with an arithmetic mean 2.84 out of 4 , where the female students of Imam Muhammad ibn Saud Islamic University were ranked the highest in agreement with an arithmetic mean 2.86 out of 4 ; followed by those of Princess Nourah bint Abddulrahman University, with 
an arithmetic mean 2.85 out of 4; and those of King Saud University, with an arithmetic mean 2.82 out of 4 .

3. "Illustrating the prohibition of self-aggression and avoiding injustice", with an arithmetic mean 2.80 out of 4 , where the female students of Imam Muhammad ibn Saud Islamic University were ranked the highest in agreement with an arithmetic mean 2.82 out of 4; followed by those of Princess Nourah bint Abddulrahman University, with an arithmetic mean 2.81 out of 4; and those of King Saud University, with an arithmetic mean 2.79 out of 4 .

4. "Children avoid the manifestation of behavioral deviation", with an arithmetic mean 2.78 out of 4 , where the female students of Imam Muhammad ibn Saud Islamic University were ranked the highest in agreement with an arithmetic mean 2.80 out of 4 ; followed by those of Princess Nourah bint Abddulrahman University, with an arithmetic mean 2.78 out of 4; and those of King Saud University, with an arithmetic mean 2.75 out of 4 .

5. "Linking children to good friends", with an arithmetic mean 2.77 out of 4 , where the female students of Imam Muhammad ibn Saud Islamic University were ranked the highest in agreement with an arithmetic mean 2.81 out of 4; followed by those of Princess Nourah bint Abddulrahman University, with an arithmetic mean 2.78 out of 4; and those of King Saud University, with an arithmetic mean 2.73 out of 4 .

The most significant role of woman in implanting the moderate Islamic values in children is to educate children about the rules and ethics of Islam, because the mother is keen on bringing up children in a moderate Islamic way and educates them the Islamic rules and ethics.

III. Results related to the third question regarding the role of woman in reinforcing and respecting the self and maintaining cultural distinctiveness, in a descending order as follows:

1. "Embracing children and dealing with them with love and understanding", with an arithmetic mean 2.69 out of 4, where the female students of Imam Muhammad ibn Saud Islamic University were ranked the highest in agreement with an arithmetic mean 2.77 out of 4; followed by those of Princess Nourah bint Abddulrahman University, with an arithmetic mean 2.69 out of 4; and those of King Saud University, with an arithmetic mean 2.60 out of 4 .

2. "Helping children take good models", with an arithmetic mean 2.68 out of 4 , where the female students of Imam Muhammad ibn Saud Islamic University were ranked the highest in agreement with an arithmetic mean 2.74 out of 4 ; followed by those of Princess Nourah bint Abddulrahman University, with an arithmetic mean 2.69 out of 4 , and those of King Saud University, with an arithmetic mean 2.61 out of 4 .

3. "Providing a family environment characterized by strong communication", with an arithmetic mean 2.68 out of 4, where the female students of Imam Muhammad ibn Saud Islamic University were ranked the highest in agreement with an arithmetic mean 2.76 out of 4; followed by those of Princess Nourah bint Abddulrahman University, with an arithmetic mean 2.68 out of 4; and those of King Saud University, with an arithmetic mean 2.59 out of 4 .

4. "Helping and motivating children to achieve success to serve community", with an arithmetic mean 2.65 out of 4, where the female students of Imam Muhammad ibn Saud Islamic University were ranked the highest in agreement with an arithmetic mean 2.72 out of 4; followed by those of Princess Nourah bint Abddulrahman University, with an arithmetic mean 2.66 out of 4; and those of King Saud University, with an arithmetic mean 2.57 out of 4 .

5. "Helping children pride themselves on boasting the culture and civilization of community", with an arithmetic mean 2.61 out of 4 , where the female students of Imam Muhammad ibn Saud Islamic University were ranked the highest in agreement with an arithmetic mean 2.66 out of 4; followed by those of Princess Nourah bint Abddulrahman University, with an arithmetic mean 2.61 out of 4 ; and those of King Saud University, with an arithmetic mean 2.55 out of 4 .

The most significant role of woman in reinforcing, respecting, and improving the self and maintaining cultural distinctiveness is embracing children and dealing with them with love and understanding because the mother always seeks to provide a sound family environment to children.

\section{$I V$. Results related to the fourth question regarding the role of woman in bringing up children on respecting freedom, in a descending order as follows:}

1. "Illustrating the prohibition of self-aggression and avoiding injustice", with an arithmetic mean 2.74 out of 4, where the female students of Imam Muhammad ibn Saud Islamic University were ranked the highest in agreement with an arithmetic mean 2.80 out of 4 ; followed by those of Princess Nourah bint Abddulrahman University, with an arithmetic mean 2.75 out of 4; and those of King Saud University, with an arithmetic mean 2.66 out of 4 .

2. "Educating children about positive treatment methods with others", with an arithmetic mean 2.72 out of 4 , where the female students of Imam Muhammad ibn Saud Islamic University were ranked the highest in agreement with an arithmetic mean 2.80 out of 4 ; followed by those of Princess Nourah bint Abddulrahman University, with an arithmetic mean 2.73 out of 4, and those of King Saud University, with an arithmetic mean 2.63 out of 4 .

3. "Guiding children not to mock and humiliate others", with an arithmetic mean 2.72 out of 4 , where the female students of Imam Muhammad ibn Saud Islamic 
University were ranked the highest in agreement with an arithmetic mean 2.79 out of 4 ; followed by those of Princess Nourah bint Abddulrahman University, with an arithmetic mean 2.73 out of 4 ; and those of King Saud University, with an arithmetic mean 2.65 out of 4 .

4. "Guiding children to benefit from positive opinions", with an arithmetic mean 2.68 out of 4 , where the female students of Imam Muhammad ibn Saud Islamic University were ranked the highest in agreement with an arithmetic mean 2.77 out of 4; followed by those of Princess Nourah bint Abddulrahman University, with an arithmetic mean 2.69 out of 4; and those of King Saud University, with an arithmetic mean 2.58 out of 4 .

5. "Helping children face situations of failure", with an arithmetic mean 2.65 out of 4 , where the female students of Imam Muhammad ibn Saud Islamic University were ranked the highest in agreement with an arithmetic mean 2.73 out of 4 ; followed by those of Princess Nourah bint Abddulrahman University, with an arithmetic mean 2.66 out of 4 ; and those of King Saud University, with an arithmetic mean 2.56 out of 4 .

The most significant roles of woman in bringing up children on respecting the freedom of others are "illustrating that Allah prohibited killing the soul except by legal right" because the mother often seeks to her children being affected with wrong ideas that disseminated lately.

\section{The Proposal of Woman's Role in Achieving Intellectual Safety in the Saudi Community}

The proposal mainly aims to activate the role of woman in achieving intellectual safety in the Saudi community so that family institutions can benefit from in educating and guiding woman about her active role in achieving intellectual safety from all aspects.

It depends on the following:

I. Limitations of the Study:

II. The proposal

III. Recommendations:

\section{Limitations of the Study: The study depends on two main} domains:

The first focuses on the study's objectives:

1. The role of woman in bringing up children according to the culture and mechanism of family dialogue.

2. The role of woman in implanting moderate Islamic values in children.

3. The role of woman in reinforcing and respecting the self and maintaining cultural distinctiveness.

4. The role of woman in bringing up children on respecting the freedom of others.

The second addresses the conditions of the population that are manifested via the general results of the study, including:

1. The most significant roles of woman in bringing up children according to the culture and mechanism of family dialogue are that she "educates children" because the mother is keen on bringing them up in a way they adopt with community by acquiring the permanent community culture.

2. The most significant role of woman in implanting moderate Islamic values in children is to educate children about the rules and ethics of Islam, because the mother is keen on bringing up children in a moderate Islamic way and educates them the Islamic rules and ethics.

3. The most significant role of woman in reinforcing, respecting, and improving the self and maintaining cultural distinctiveness is embracing children and dealing with them with love and understanding because the mother always seeks to provide a sound family environment to children.

4. The most significant roles of woman in bringing up children on respecting the freedom of others are "illustrating that Allah prohibited killing the soul except by legal right" because the mother often seeks that her children being affected with wrong ideas that disseminated lately.

Accordingly, the study's proposal is introduced.

\section{The proposal of woman's role in achieving intellectual safety in the Saudi community}

(1) Beneficiaries

- Ministry of Social Affairs

- Ministry of the Interior

- Middle and Secondary Education Sector

- Saudi Universities and Higher Education Sector

Hence, it can be formulated, as follows:

1. A preventive aspect

2. A remedial aspect

The preventive aspect is represented in

1. The importance of proposing long and short term plans that contribute to activating the role of woman and family in achieving intellectual safety, according to:

- Forming a committee of consultants and specialists in advising, family counseling, social service, and criminology to consider setting the required plans.

- Forming a committee of the directors of the institutions related to family and woman to address the related problems and difficulties regarding the role of woman in achieving intellectual safety and suggesting solutions.

It is suggested that the content of the strategic preventive plan should focus on:

1. Awareness programs to families to educate parents, especially the mothers, about the sound educational methods that help promote children's personalities, familiarize them with opinions' exchange and avoid fanaticism, disseminating cruelty and violence 
2. Social and environmental determinants are the most contributing ones in the emergence of intellectual extremism due to awareness programs (lectures, workshops, brochures, and movies) to educate the young and reject extremism.

3. Socialization institutions should be interested in the preventive programs to youth and adolescents to enlighten them and help accept the opinions of others and effective dialogue to assure that they will not have intellectual extremism.

4. Conducting preventive programs to youth and adolescents in families, schools, and universities to help students entertain nationalism and educate families about the importance of mental education to children.

5. On designing the preventive programs, it should be taken into consideration implying student activities in schools and universities to upgrade intellectual awareness and help them freely express their opinions, ideas, and needs.

6. Clarity of the role of mosque, university, and family regarding the concept of religiousness and unifying resources to agree on moderation and rejecting intellectual extremism.

7. Creating preventive programs to be implemented in the classrooms in all stages, the most important of which are (disseminating the culture of dialogue among students, respecting freedoms, and nationalism to familiarize children with this culture and protect them from dangerous situations or involvement in terrorist acts.

8. Service-learning has a great impact in reinforcing students' learning; giving them knowledge, values, attitudes, skills, and experiences based on the community and its problems; and enlightening them of the reality of community.

9. Coordination and cooperation among Ministries of the interior, Social Affairs, Education, and Higher Education by their representatives to set the strategic plan and define implementation techniques.

The remedial aspect is represented in

1. The importance of discovering students in the various educational stages who manifest some forms of intellectual extremism in addition to designing social, religious, and educational remedial programs supervised by specialists in all aspects to help change and modify students' ideas and attitudes.

2. The importance of forming a sub-unit in each socialization institution, entitled "Intellectual Awareness Unit" that is concerned with providing preventive and remedial programs and hires specialists to correct attitudes and ideas to achieve intellectual safety.

3. Using role play technique with adolescents and youth in schools and universities who manifest some forms of intellectual extremism to help represent some roles of intellectually extremist. They should also be consulted to define the required remedial procedures.

4. The role of media in achieving intellectual safety cannot be ignored. Accordingly, the strategic plan should include the role of media in highlighting the role of mother in the effective family dialogue, sound parental treatment, and moderation. This is reflected in their personality and current and future behaviors.

The proposal can be summarized, as follows:

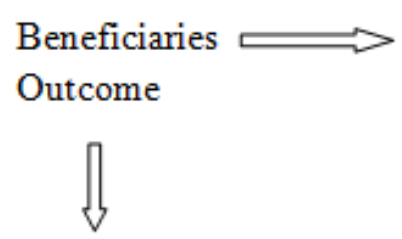

1. Concemed ministries Achieving

- Interior intellectual safety

- Social Affairs

- Education

- Youth

- Higher Education

on using scientific strategies, methods, and techniques.

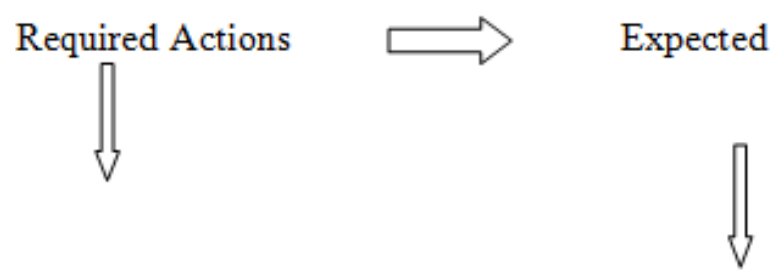

A strategic plan that covers

- A preventive aspect

- A remedial aspect

implemented by socialization

institutions (i.e. family/school/ university

mosque/ educational institutions) and depends 


\section{Recommendations:}

The following recommendations are made:

1. Activating the role of families and educational institutions in enlightening children of all ages and stages to reject extremism and providing a social environment free from intellectual extremism, using dialogue and exchange of opinions.

2. Activating the role of media in designing awareness programs to parents to shed light on the sound educational methods that should be used with children to educate them according to the Islamic socialization through setting cultural and educational programs to limit the negative aspects of globalization.

3. Offering awareness programs to children to shed light on the types of dangerous situations that may face them and cause their involvement in works that conflict with values and intellectual safety; they should be provided with methods that help entertain protection and intellectual awareness.

4. Holding symposia, scientific conferences, and meetings to discuss the issues related to the role of mother in achieving intellectual safety in the family, the difficulties that may affect, and the mechanism of activation in a way that achieves intellectual safety of the children and community.

5. Interest in activities that may raise intellectual belonging by activating administrative control to assure that all activities work well to achieve intellectual safety in the various institutions.

6. Activating the role of supervision and academic and student advising in Saudi universities and schools to consolidate the concept of nationalism and change and modify the wrong ideas about the community among such youth.

7. Designing awareness programs to families, and mothers, in particular, focusing on the way, mechanism, and importance of sound Islamic education to families.

8. The mosque and religious discourse play an effective role in defining, describing, and activating the mother's role in achieving intellectual safety to children.

9. Consolidating the role of the school and mother in protecting children from the falls of intellectual deviation and helping them achieve intellectual safety.

10. Educating families about family dialogue and disseminating this culture in the Saudi community through preventive programs by the mass media, educational and family institutions, and the various socialization ones such as the mosques.

\section{REFERENCES}

[1] Elashqer, M. N. (2010). The role of student activities in consolidating intellectual safety: Towards creating an intellectual sample to consolidate intellectual safety (unpublished Ph.D. dissertation). Riyadh: Naif Arab University for Security Sciences.

[2] Elbaqmey,S. S. (2010). Towards creating the project of intellectual safety reinforcement, Ministry of Education. Paper presented at the first national conference of intellectual safety "Concepts and Challenges".

[3] Elbashry, M. A. (2000). Arab security: Foundations and obstacles. Riyadh: Naif Arab University for Security Sciences.

[4] Eldeaj, F. A. (1985). Security and media in the Islamic state. Riyadh: Publishing house of the Arab center of security studies and training.

[5] Eljahny, A. F. (2004). Family role in consolidating intellectual security. Journal of Police Thinking, 12, 4.

[6] Elhaider, H. A. (2002). Intellectual safety in the face of intellectual impacts (unpublished Ph.D. dissertation). Cairo: Cairo University.

[7] Elhazemy, Z. Z. (2010). National dialogue and its role in reinforcing national security of KSA (3rd ed.). Riyadh: King Abdul Aziz Centre for National Dialogue.

[8] Elrashedy, M. R. (2012). Psychological and environmental determinants of intellectual extremism among youth as perceived by the faculty members of public universities in Riyadh (Unpublished MA. thesis).

[9] Elshahrany, S. A. (2005). Intellectual deviation and its impact on the national and social safety of the Cooperation Council for the Arab States of the Gulf. Riyadh: Naif Arab University for Security Sciences.

[10] Elsaleh, M. (2004). Social control ( $1^{\text {st }}$ ed.). Dar Elwaraq.

[11] Elhawaish, Y. (2011). Reinforcing intellectual safety among high school students in the light of the contemporary global models of dialogue, a proposal. Unpublished $\mathrm{Ph}$. D thesis in Fundamental of Education.

[12] Ameen, H. A. (2012). Consolidating the role of woman in education, implanting positive ideas, and reinforcing nationalism of children and youth to counter terrorism. Paper presented at the second conference "impact of terrorism on social development". Riyadh: Naif Arab University for Security Sciences.

[13] Conrad, D. (1995). High school community service review of research and programs. Washington, DC: Office of Educational Research and Improvement ERIC no: 313569.

[14] Egan, A. (2007). Dressing up terrorism: A media analysis of women's involvement in terrorism (MA thesis). Alberta: Calgary University.

[15] Greenword, P. (1996). The cost-effectiveness of early intervention as a strategy for reducing violent grime, Santa Monica: RAND.

[16] Grahmj \& Bowling, B. (1998). Young people and crim. Home office, research and statistics department.

[17] Katsos, T. (2003). Teaching in troubled times: A study of September 11 and its impact on schooling.

[18] Yahyaei, D. \& Mosadeghzadeh, H. (2014). The role of women in promoting family mental security. Indian Journal of Fundamental and Applied Life Sciences, 4 (4), PP. 2325-2333. 
[19] Elqadeb, M. (2010). The Role of High School Principals in Achieving Intellectual Security in Riyadh, Unpublished Master Thesis, Department of Educational Administration, College of Education, King Saud University.
[20] Alotaibi, A. (2007). The Role of Women in Enhancing Intellectual Security among High School Students, Unpublished Master Thesis, College of Education, King Saud University. i This paper is funded by The Center for Promising Research in Social
Studies and Women's at Princess Nourah bint Abdulrahman University,
Saudi Arabia (3709) 